\title{
Development and benchmarking of Open Force Field v1.0.0, the Parsley small molecule force field
}

4 Yudong Qiu ${ }^{1}$, Daniel G. A. Smith ${ }^{2}$, Simon Boothroyd ${ }^{3}$, Hyesu Jang ${ }^{1}$, Jeffrey Wagner ${ }^{4}$, Caitlin C. Bannan ${ }^{4,8}$, Trevor Gokey4 , Victoria T. Lim ${ }^{4}$, Chaya D. Stern ${ }^{3}$, Andrea Rizzi ${ }^{3,9}$, Xavier Lucas ${ }^{5}$, Bryon Tjanaka ${ }^{4}$, Michael R. Shirts $^{6}$, Michael K. Gilson ${ }^{7}$, John D. Chodera ${ }^{3}$, Christopher I. Bayly ${ }^{8}$, David L. Mobley ${ }^{4}$, Lee-Ping Wang ${ }^{1}$

$8 \quad{ }^{1}$ Chemistry Department, The University of California at Davis, Davis, California 95616; ${ }^{2}$ The - Molecular Sciences Software Institute (MolSSI), Blacksburg, VA 24060; ${ }^{3}$ Computational \&

Abstract We describe the structure and optimization of the Open Force Field 1.0.0 small molecule force field, code-named Parsley. Parsley uses the SMIRKS-native Open Force Field (SMIRNOFF) parameter assignment formalism in which parameter types are assigned directly by chemical perception, in contrast to traditional atom type-based approaches. This method provides a natural means to incorporate increasingly diverse chemistry without needlessly increasing force field complexity. In this work, we present essentially a full optimization of the valence parameters in the force field. The optimization was carried out with the ForceBalance tool and was informed by reference quantum chemical data that include torsion potential energy profiles, optimized gas-phase structures, and vibrational frequencies. These data were computed and are maintained with QCArchive, an open-source and freely available distributed computing and database software ecosystem. Tests of the resulting force field against compounds and data types outside the training set show improvements in optimized geometries and conformational energetics and demonstrate that Parsley's accuracy for liquid properties is similar to that of other general force fields. 


\section{Introduction}

Molecular mechanics (MM) force fields are empirical models of molecular potential energy surfaces, which yield the potential energy and atomic forces as a function of the atomic positions. Force fields are a crucial component of molecular simulations in many domains of chemistry and biophysics. They are widely used to analyze biomolecular systems that may include biopolymers, aqueous solvent, and small molecules such as metabolites and therapeutics. They are also fundamental to technologies used in computer-aided drug design, such as molecular docking[1-7] and simulation-based calculations of protein-ligand binding free energies[8-15].

Decades of work have led to relatively refined force fields for proteins made up of the 20 common amino acids[16-24]. However, it is more difficult to develop a high quality general force field, i.e., one that applies to the wide range of small, organic molecules of interest in biology and drug discovery, due to the high diversity of the chemical space that must be considered. For example, the nearly 100 million compounds in the PubChem database[25] embody many different combinations of varied functional groups and heterocycles. Moreover, some of the most important applications of force fields involve the simulation of as-yet undiscovered compounds, such as those under investigation for small molecule drug development, which may lie in new regions of chemical space. Small molecule force fields in wide use today include the general AMBER force field (GAFF),[26] the CHARMM general force field (CGenFF),[27] and the optimized potentials for liquid simulations force field (OPLS).[28] These important tools have undergone continuous development, and current generations are applicable to a wide range of small molecules. Nonetheless, recent calculations of hydration free energies, partition coefficients, and other properties show clear systematic errors for specific functional groups in current general force fields [29-32]. Therefore, there is a need to improve small molecule force fields. At the same time, our ability to advance force field accuracy continues to increase, as more data become available from experiments and high-level theoretical calculations, and greater computer power enables systematic and comprehensive approaches to parameterization.

For many years, the standard use of atom-typing as a first step in parameter assignment has posed a barrier to the facile development of general force fields. In the first step of atom typing, chemical perception (i.e. substructure searching) is first used to assign an atom type to each atom in the molecule based on its local chemical environment, e.g. " $s p^{2}$ carbon double bonded to oxygen". The atom types are then combined into keys that are used to look up parameters in a table, e.g. Lennard-Jones parameters are indexed by a single atom type whereas angle parameters are indexed by three atom types. This atom typing formalism has been mostly unchanged since its inception, which attests to its simplicity and usefulness. However, as previously detailed,[33] it drives a needless level of complexity in the force field specification. For example, if new QM data lead the developer to add a new dihedral type, specifying this may require addition of another atom type; but this in turn requires adding parameters for all the FF terms that involve this type, such as Lennard-Jones, bond-stretch, and angle-bend. These complexities have made it difficult to systematically modify force fields and extend them to new chemistries. 


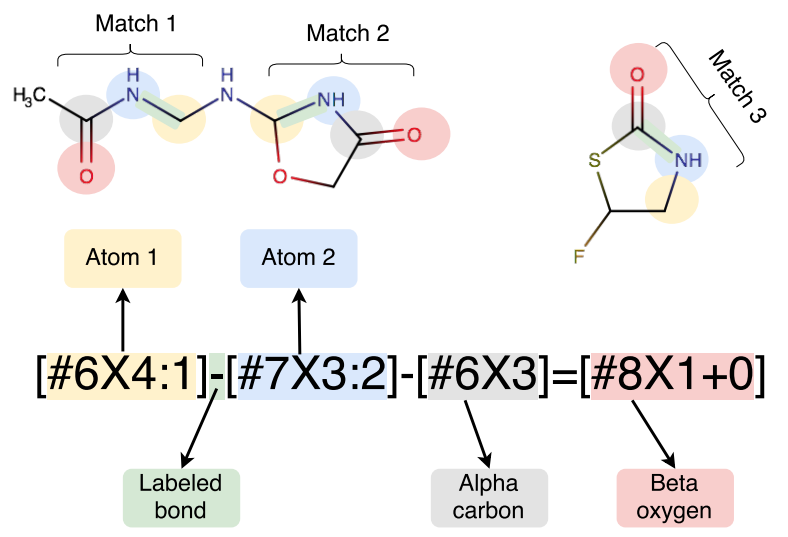

Figure 1. Parameter typing for a 2-body interaction using SMIRKS strings. The string matches a carbon with valence 4 \#6x4 tagged "1", bonded to a nitrogen with valence 3 \#7 33 tagged "2", bonded to a carbon with valence 3 \#6x3, double bonded to a neutral oxygen with valence $1 \# 8 \times 1$. The parameters attached to the SMIRKS string (for example, $r_{0}=$ $1.5 \AA, k=400 \mathrm{kcal} \mathrm{mol}^{-1} \AA^{-2}$ ) are assigned to the interaction between atoms tagged " 1 " and "2".

Instead of using atom-typing, we use the SMIRKS Native Open Force Field (SMIRNOFF) formalism, which assigns parameters by what we call direct chemical pattern perception, via substructure searches [33]; see Fig 1. In SMIRNOFF, the parameter definitions for each separate force field term, such as van der Waals $(\mathrm{vdW})$, bond stretching, angle bending, or torsion, are defined by a hierarchy of increasingly specific substructure rules, where each rule consists of a SMIRKS pattern attached to numerical parameters. When a SMIRKS pattern is matched to a chemical substructure in a molecule, the attached parameters are assigned to the tagged atoms within the substructure (Figure 1). The critical distinction from atom typing is that here, each force field term has its own hierarchy, so its rules can be adjusted, and new rules can be added, without perturbing the rules used for the other terms. This formalism is implemented using the Open Force Field toolkit, an open-source software package that allows for the parameterization of general small molecules.

The recent development of the SMIRNOFF99Frosst [33] force field proved the concept of the SMIRNOFF typing formalism. The types and numerical parameters of SMIRNOFF99Frosst were designed for maximal consistency with the Parm@Frosst force field[34], a general force field in the AMBER family with similarities to GAFF, and indeed, SMIRNOFF99Frosst retained the accuracy of simulations for several important properties, such as the densities and dielectric constants of organic liquids, hydration free energies of small molecules, and host-guest binding thermodynamics.[35] Importantly, the new typing formalism allowed this to be achieved with only $~ 300$ lines of parameters, which may be compared with $>6000$ lines of parameters for GAFF. Moreover, the independent hierarchies of typing rules for each force field term allows for simple extensions to increase accuracy in new areas of chemical space. This prior work sets the stage for optimizing the parameter sets using available and/or newly generated training data from quantum chemistry and/or experiment.

Here, we describe the development of OpenFF 1.0.0, code-named Parsley, the first optimized force field using the SMIRNOFF format. To create Parsley, we reoptimized nearly all (481/500) of the valence parameters against quantum chemical data, leading to improved agreement with quantum chemical geometries, energetics, and vibrational frequencies. Parsley largely retains the nonbonded interactions of SMIRNOFF99Frosst; it uses the same Lennard-Jones parameters, with a few changes to the Lennard-Jones typing hierarchy, and continues to use the AM1-BCC [36] charge model. The resulting force field provides improved accuracy relative to its predecessor for a wide variety of properties, especially energetics and geometries relative to gas phase quantum chemical calculations. In keeping with the Open Force Field Initiative's core philosophy, the Parsley force field, and the software tools and data sets created during the development of Parsley, are released under permissive open source licenses. These research products, along with some existing software tools used here, are also available on an open and free basis, with some limited exceptions, notably that commercial OpenEye toolkits (which are free for academics) were used in portions of the procedure. 
We also report here on the initial benchmarking of Parsley, which focuses on two major aspects: (1) accuracy relative to quantum chemical calculations on small molecules in the gas phase; and (2) accuracy of liquid-state properties for a diverse set of organic molecules. For the condensed-phase properties, which span density, dielectric constant, heat of vaporization, excess molar volume, and enthalpy of mixing, no dramatic performance differences were noted in this release, relative to SMIRNOFF99Frosst, and the overall accuracy is similar to that of GAFF. This is as expected, because valence parameters typically play a relatively minor role in these properties. However, the quantum chemical benchmarks, which cover more than 2,000 molecules and consider the quality of optimized geometries and relative conformer energies, show substantially better performance relative to SMIRNOFF99Frosst. The present Parsley force field represents the first of what we expect will be a series of continually improving, open force fields for small molecules.

\section{Methods}

The Parsley force field was generated by refitting the parameters in the valence terms of SMIRNOFF99Frosst [33] against an extensive new set of high-level quantum mechanical data, which include energies, gas-phase geometries, and other properties. Note that the starting parameters in SMIRNOFF99Frosst in turn originated from two parent force fields, AMBER parm99[37] and Merck's parm@Frosst[34], which had been parameterized to reproduce gas phase geometries and energetics computed at lower levels of QM than that used here, for selected molecules. Here, Section 2.1 details the force field parameters that were optimized, the QM dataset used to drive the optimization, and the application of ForceBalance [38] to carry out the optimization from the SMIRNOFF99Frosst [33] starting point. Section 2.2 then describes how the resulting Parsley force field was tested against gas phase QM data and condensed phase data outside the training sets.

\subsection{Training the Parsley force field}

\subsubsection{Parameters that were refit}

We reoptimized most of the valence parameters present in SMIRNOFF99Frosst. In the following discussions, an individual parameter definition is uniquely identified by an interaction type (e.g. bond stretch) and a SMIRKS pattern (e.g. [\#6X4:1] - [\#6X4:2]), and can contain one or more physical values (e.g. the bond length and the force constant). The full list of parameter definitions, which can be viewed in the published force field XML file, openff-1.0.0-RC2. of fxm1[39], may be summarized as follows:

- Harmonic bond stretch: 86 equilibrium bond lengths and force constants.

- Harmonic angle bend: 35 equilibrium angles and 39 force constants. These two numbers differ because four angles are linear and were kept linear during fitting.

- Proper torsions: Each of the 154 torsion types is associated with an $\mathrm{N}$-term Fourier series of potential energy contributions, where $N \leq 6$, and each term, $i$, is of the form $E_{i}=k_{i} \cos \left(i \phi+\delta_{i}\right)$. We optimized all of the amplitudes that were defined in SMIRNOFF99Frosst, comprising 154,62, 26, 5, 4 and 3 values of $k_{1}, k_{2}, k_{3}, k_{4}, k_{5}$, and $k_{6}$ respectively, for a total of 254 parameters. Parameters $\mathrm{t} 156, \mathrm{t} 157, \mathrm{t} 158$ represent torsion angles containing a linear angle, and their values of $k_{1}$ were kept at 0.0 during fitting. The phase parameters, $\delta_{i}$ and the selection of Fourier terms used for each torsion were not optimized in this release.

- Improper torsions: The four improper terms were kept unmodified, to avoid overfitting.

Thus, we were prepared to fit up to 500 bonded parameters (applied by 279 SMIRKS patterns: 86 bonds, 39 angles, and 154 torsions). However, the quantum chemical datasets we utilized for fitting (Section 2.1.2) exercised only 481 of these parameters, so 19 parameters were left unchanged here. All of the above parameters were fitted simultaneously against all QM data.

\subsubsection{Compound sets used in training}

Two sets of small organic molecules were used to generate the quantum chemical datasets used in fitting. The first, termed the Roche Set, contains 468 fragment-sized molecules, most containing one to three rotatable bonds, that were provided by Roche as a collection of important and/or interesting chemistries. 
Representative compounds from this set are illustrated in Figure 2, and the full set can be found in Supporting Information section 1.1.1. The second, termed the Coverage Set, contains 80 molecules selected from eMolecules[40] using a greedy algorithm aimed at providing parameter coverage for the maximum number of parameters using the minimum number of molecules. Figure 3 illustrates representative compounds, and a full list of SMILES can be found in Supporting Information section 1.1.1. 


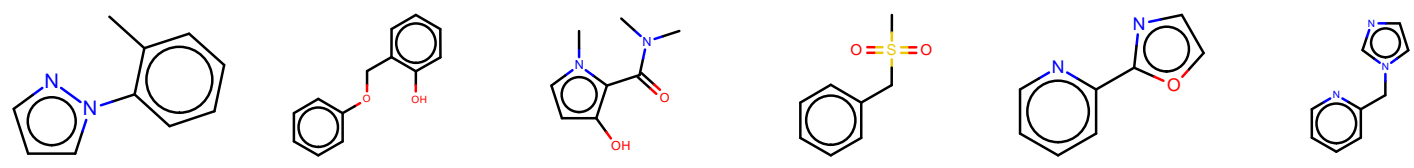

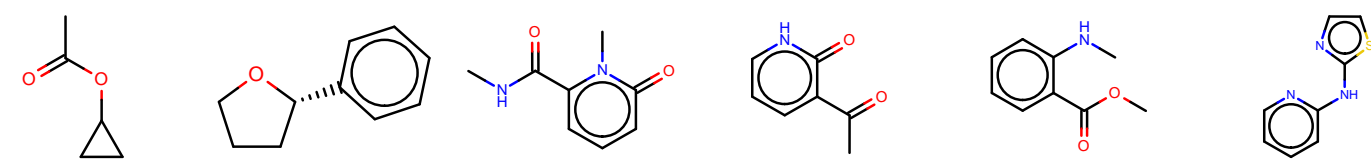

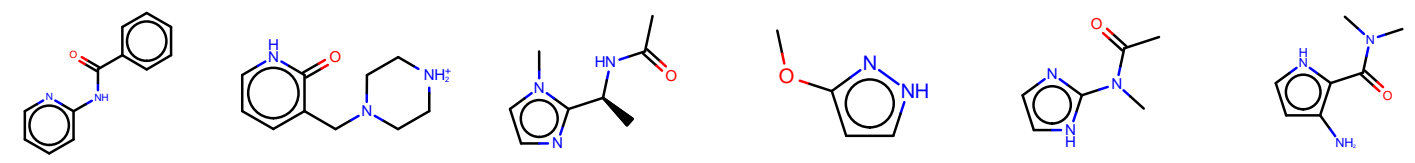

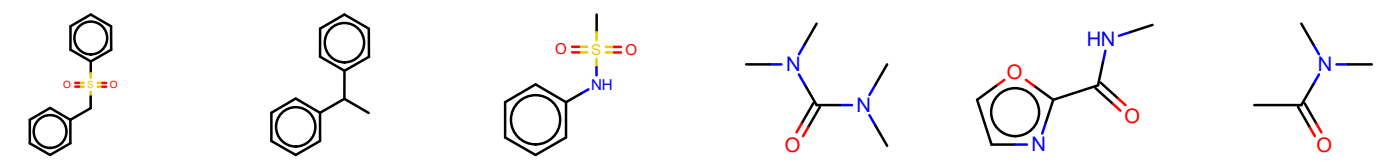<smiles>O=c1cccnn1-c1ccccc1</smiles><smiles>CCc1ccccc1CC</smiles><smiles>C/C=C/CC</smiles><smiles>CC(c1ccccc1)C(C)(C)C</smiles><smiles>NC(=O)c1ccccc1N</smiles><smiles>CCCC(=O)C(C)C</smiles><smiles>CCC(=O)C(=O)C(C)C</smiles><smiles>CNC(=O)c1c(O)ccn1C</smiles><smiles>FCOc1ccccc1</smiles><smiles>CC(=O)c1ccoc1C(C)=O</smiles><smiles>c1ccc(C2CCCCC2)cc1</smiles><smiles>CC(=O)Cc1ccccc1I</smiles>

Figure 2. An illustrative subset of small fragment-like molecules from the Roche Set. 

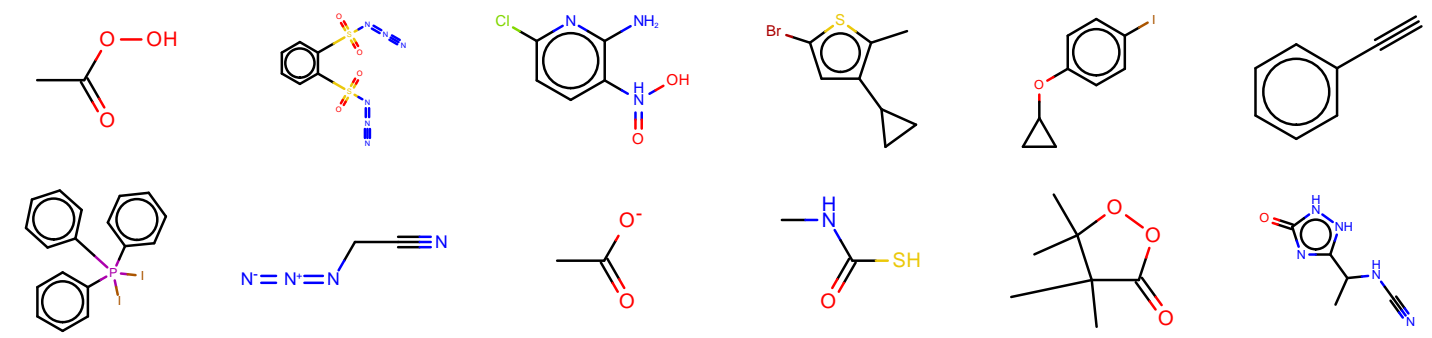<smiles>CC(=O)[O-]</smiles>
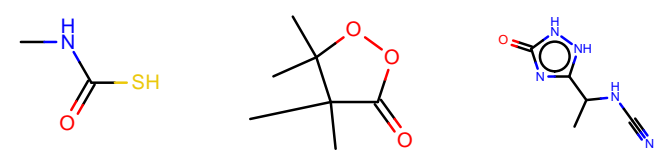<smiles>NNCc1cnns1</smiles><smiles>CCc1ccc(N=S=O)cc1</smiles><smiles>OC(O)(O)O</smiles><smiles>CNC(=O)NCOC</smiles><smiles>O=C(CC1CC(O)CS1)NC1CC1</smiles><smiles>CC(C)c1ccc(I)cc1</smiles><smiles>CC(C)(c1ccccc1)c1ccccc1</smiles><smiles>CCCc1ccccc1</smiles><smiles>CC(C)(C)C(C)(C)C(C)(C)C(C)(C)C</smiles><smiles>CNN1CC(C)CS1(=O)=O</smiles><smiles></smiles><smiles>C[SH](N)(=O)NC1CC1</smiles><smiles>Cc1cccc(I)c1</smiles><smiles>CC(=O)OC1CC1OC(C)=O</smiles><smiles>CCC(C)(C)c1ccc(I)cc1</smiles><smiles>CC1=CC(=O)N(C)S1(=O)=O</smiles><smiles>C[C@@H]1CNC[C@H]1C</smiles><smiles>[B]C([B])[B]</smiles><smiles>C[As](C)(=O)O</smiles><smiles>CS(=O)Cl</smiles><smiles>CC(=O)C1=CC=C[C@@H]1C</smiles><smiles>CC1(C)CC1(C(N)=O)C(=O)O</smiles><smiles>CC1(O)C2CCCC21</smiles><smiles>Cc1ccc(S(=O)(=O)I)cc1</smiles>

Figure 3. An illustrative subset of molecules from the Coverage Set.

Initial automated selection of the Coverage Set is described in a subdirectory of the openforcefields GitHub repository, and details of the additional molecules added manually to cover remaining gaps can be found in Supporting Information section 1.1.1. Nonetheless, for a variety of technical reasons, it proved difficult to generate compounds with suitable quantum chemical data to cover 19 parameters. In future 
work, these gaps will need to be addressed by generation of suitable data and/or deletion of parameter types that may be overly specialized or exotic.

\subsubsection{Selection of quantum chemistry methodology}

Quantum chemical calculations (geometry optimizations and torsion scans) were performed on a distributed set of high-performance computing clusters using the MoISSI QCFractal[41] distributed quantum chemistry engine, with results deposited in the public MoISSI QCArchive Server (MQCAS)[42, 43] to allow open public access to all data. We used a single level of theory for all QM calculations, B3LYP-D3(BJ) / DZVP [44-47]. This choice was based on two benchmark studies of conformational energies $[48,49]$ and our own initial studies that aimed to balance accuracy against computational cost. The molecules in both of these studies included amino acids, small to medium-sized peptides, and macrocycles. Geometries were optimized at the MP2/ccpVTZ level, and reference energies were computed using explicitly correlated focal point analysis methods considered to be equivalent to complete basis $\operatorname{CCSD}(\mathrm{T})$ in accuracy. Both studies found that B3LYP-D3(BJ) reproduces the reference energies with RMSEs of $<1 \mathrm{kcal} / \mathrm{mol}$ when very large basis sets (e.g.def2-QZVP [50]) are used; the empirical D3 dispersion term played a major role, as the errors were typically $2-4 \mathrm{x}$ larger without it.

Notably, Řezáč et al. 2018 [48] reported that the double-zeta quality DZVP basis set [44] gave nearly the same RMSE as def2-QZVP, which we were able to reproduce in our own tests. When similar-sized and better-known basis sets such as 6-31G* and def2-SV(P) were used, the RMSEs increased significantly but there were only minor differences in computational cost. Our results are largely consistent with Řezáč et al. 2018 [48] even though we did not use the custom empirical dispersion parameters they derived for the DZVP-DFT basis set. A scatter plot of RMSE vs. calculation time for a representative molecule, labeled as FGG114 in Řezáč et al. 2018.[48], is shown in Figure 4; the results confirm that the DZVP-DFT basis set gives the best compromise between accuracy and computational cost.

Although we believe our choice of QM method is appropriate for gas-phase conformational energies for the neutral compounds comprising our training set here, we did not conduct benchmark studies on optimized geometries and vibrational frequencies which were also part of our parameterization dataset. More comprehensive benchmarks are planned to inform future force field generations. However, the present level of theory is superior to the HF/6-31G* approach used in parameterizing the parm99/parm@Frosst force fields from which SMIRNOFF99Frosst descended, and thus should afford greater accuracy.

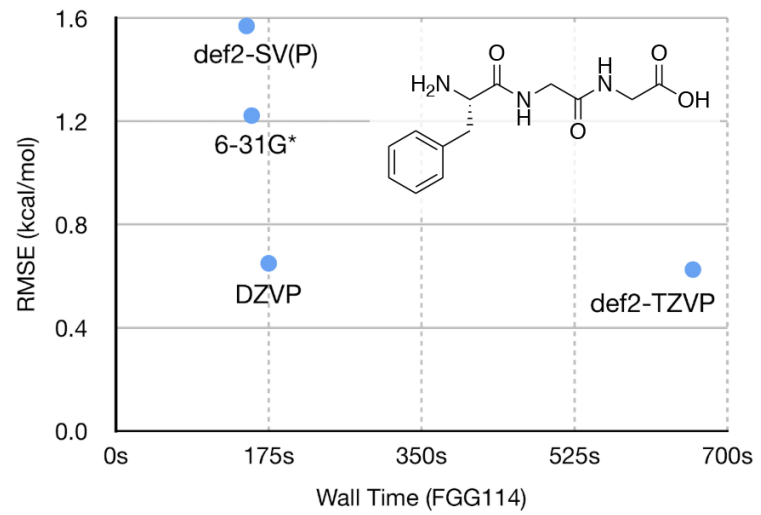

Figure 4. Tradeoff between speed and accuracy in selecting quantum chemical basis set. Computational time (for single conformer) versus RMSE to benchmark-quality relative energies for 15 conformations of a representative molecule from Ref. 48 for several choices of basis set.

\subsubsection{Generation of quantum chemical data for compound datasets}

Prior to running quantum chemical calculations, the input molecules were subjected to protonation state and conformer expansion, using the Fragmenter software package.[51] After the expansion, each protonation state was identified as a new molecule, so the number of distinct molecules increased; and each 


\begin{tabular}{|l|l|c|c|}
\hline & & Roche Set & Coverage Set \\
\hline & Compounds & 468 & 80 \\
\hline & Cmpds $\times$ Prot. States & 468 & 233 \\
\hline \multirow{2}{*}{ Opt. Geom. } & Cases & 936 & 831 \\
\cline { 2 - 4 } & MQCAS Dataset & OpenFF Optimization Set 1 & SMIRNOFF Coverage Set 1 \\
\hline \multirow{2}{*}{ Vib. Freq. } & Cases & 660 & 235 \\
\cline { 2 - 4 } & MQCAS Dataset & OpenFF Optimization Set 1 & SMIRNOFF Coverage Set 1 \\
\hline \multirow{2}{*}{ Tors. Scans } & Cases & 669 & 417 \\
\cline { 2 - 4 } & MQCAS Dataset & OpenFF Group 1 Torsions & SMIRNOFF Coverage Torsion Set 1 \\
\hline
\end{tabular}

Table 1. Summary of quantum chemical calculations used to fit the force field valence parameters in this work.

molecule could have one or more conformers. Each conformer provided one optimized geometry used in fitting. Three classes of gas phase quantum chemical data were generated for both the Roche and Coverage compound sets: optimized geometries, vibrational frequencies, and torsional energy profiles. The methods used are detailed below. The results of all quantum chemical calculations are stored as DataSet objects on the MQCAS[42] and are freely available to the public. Examples of working with several MQCAS datasets can be found in Supporting Information section 1.1.2.

\section{Optimized geometries}

We used the MQCAS to store and distribute geometry optimizations with the geomeTRIC optimization driver[52] and the Psi4 quantum chemistry package[53, 54] as backends. Optimized QM geometries were downloaded from the MQCAS, then filtered to remove cases where the bonding pattern changed on optimization, as well as issues which pose other problems for the openforcefield toolkit v0.4.1[55], e.g. undefined stereochemistry, missing torsion terms, or inability to assign AM1-BCC charges. Details can be found in Supporting Information section 1.1.2.

The objective function that measures deviations of MM from QM geometries is designed in the following way: MM geometry optimizations are first locally minimized starting from QM optimized structures, then MM and QM Cartesian coordinates are converted to lists of bond lengths, bond angles, and both proper and improper torsion angles. The difference between QM and MM optimized internal coordinates for a single molecule contributes to the objective function as:

$$
L_{\text {optgeo }}(\theta)=\sum_{i \in \mathrm{ICS}}\left(\frac{x_{i}^{\mathrm{MM}}(\theta)-x_{i}^{\mathrm{QM}}}{d_{i}}\right)^{2}
$$

where $\theta$ stands for the force field parameters used in the MM calculation, $d_{i}$ refers to scaling factors of 0.05 $\AA, 8$ degrees and 20 degrees for bond lengths, bond angles, and improper torsion angles, respectively. Proper torsion angles were not considered here, but instead are fitted based on comprehensive torsional energy profiles, as detailed below.

\section{Vibrational frequencies}

For each optimized geometry in the Roche and Coverage molecule sets, Hessian calculations were both executed and stored in the MQCAS. From the calculations that were completed, the Hessians for the lowestenergy conformation of each compound / protonation state were kept. After screening the dataset for topology changes and other errors, normal mode analysis was performed to obtain harmonic vibrational frequencies and Cartesian displacements for the internal degrees of freedom. Details can be found in Supporting Information section 1.1.2.

The corresponding force field Hessians were computed by locally minimizing the QM geometries with the force field, followed by evaluating forces with numerical displacements $(0.001 \AA)$. Normal mode analysis was carried out and the QM and FF frequencies were sorted from lowest to highest to yield the sorted sequences $v_{Q M, i}$ and $v_{F F, i}$ respectively. The objective function contribution for each set of normal modes was computed 
as the sum of squared differences of corresponding frequencies, scaled by a factor of $d_{\mathrm{vib}}=200 \mathrm{~cm}^{-1}$, as:

$$
L_{\mathrm{vib}}(\theta)=\sum_{i}\left(\frac{v_{Q M, i}-v_{F F, i}}{d_{\mathrm{vib}}}\right)^{2}
$$

\subsubsection{Torsional potentials}
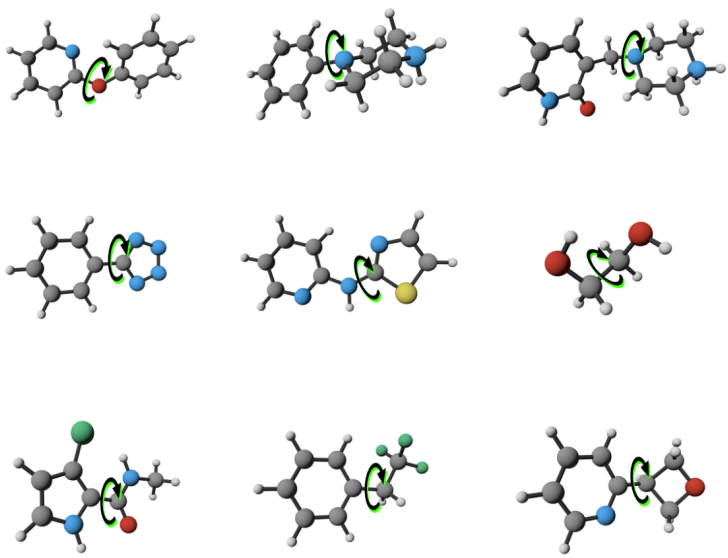

Figure 5. Example torsions selected for $1 \mathrm{D}$ torsion scans in the Roche TorsionDrive dataset. $\mathrm{H}$, white; C, gray; $\mathrm{N}$, blue; O, red; S, yellow; F, green (lower middle); Cl, green (lower left).

Quantum mechanical energy profiles were generated for dihedral angles in the Roche and Coverage sets. All calculations were carried out on the MQCAS, which employs the TorsionDrive software to compute each torsion energy profile using a wavefront propagation procedure, [56] described briefly here. Multiple initial geometries were generated for each molecule via fragmenter and provided as input at the start of the procedure. Each input structure was energy-minimized with the selected torsion angle constrained to values on a $15^{\circ}$ resolution grid, with QCArchive managing parallel job execution, and individual constrained optimizations handled by geomeTRIC/Psi4 as described above. At the conclusion of the constrained minimizations, the lowest-energy structure at each grid point was used to initiate new constrained minimizations at neighboring grid points. This cycle was repeated until the grid was fully populated with constrained minimization results and no new lowest-energy structures were found. In order to avoid pathologies such as bond-breaking that may occur when driving torsions into sterically hindered or high-energy regions, an upper energy limit was applied such that no constrained minimizations were started from structures with energies greater than $0.05 \mathrm{Hartrees}(31.3 \mathrm{kcal} / \mathrm{mol})$ above the minimum.

The set of lowest-energy constrained minimized structures for each grid point was downloaded from the MQCAS and checked for bonding topology changes; calculations that contained such changes were discarded. In addition, any scans that included a frame with an internal hydrogen bond were discarded to avoid building strong internal electrostatic interactions into fitted torsional profiles. Hydrogen bonds were detected using the Baker Hubbard method (Angle(D-H..A) $>120$ degrees and Dist $(H . . A)<2.5 \AA$ ), as implemented in the MDTraj package[57]. Details can be found in Supporting Information section 1.1.2.

For compounds in the Roche Set, torsional scans were generated for the 819 dihedral angles matching all of the following conditions:

1. the center bond is not part of a ring;

2. there is at least one heavy side group on both sides of the bond;

3. neither of the two angles involved is close to linear $\left(\geq 165^{\circ}\right)$.

Among all torsions sharing the same center bond, the one with the largest side groups, by number of atoms, was picked. For the compounds in the Coverage Set, we used the SMIRNOFF force field to label the torsions in each molecule and selected the first five dihedral angles that match each torsion term for scanning. (Note, 
however, that the force field term $t 155 \mathrm{~b}$ was added after this dataset was created, so no torsion was selected for that term.)

The objective function contribution was computed as a weighted sum of squared differences between QM and MM energies. During the fitting process, each structure along the QM torsional profile was partially relaxed using the empirical force field being optimized. These MM optimizations were started from the QM constrained optimized structure, the four atoms defining the torsion were fixed at the QM coordinates, and all other atoms were held near the QM coordinates by applying harmonic energy restraints with force constant $1 \mathrm{kcal} / \mathrm{mol} / \mathrm{A}^{2}$. These added harmonic restraints avoid the possibility of large structural changes of the MM structures away from the QM structures, which could make the torsional profile differences less meaningful. The QM and MM energies being compared were calculated as:

$$
\begin{aligned}
E_{\mathrm{QM}}\left(\mathbf{x}_{i}\right) & =E_{\mathrm{QM}}{ }^{\prime}\left(\mathbf{x}_{i}\right)-E_{\mathrm{QM}}{ }^{\prime}\left(\mathbf{x}_{0}\right) \\
E_{\mathrm{MM}}\left(\mathbf{x}_{i} ; \theta\right) & =E_{\mathrm{MM}}\left(\operatorname{OptMM}\left(\mathbf{x}_{i} ; \theta\right)\right)-E_{\mathrm{MM}}{ }^{\prime}\left(\operatorname{OptMM}\left(\mathbf{x}_{0} ; \theta\right)\right)
\end{aligned}
$$

where the primes indicate absolute energies, subscripts indicate grid point indices, $\mathbf{x}_{\mathbf{0}}$ is the lowest energy energy-minimized structure, $\theta$ represents the MM force field parameters, and OptMM(x; $\theta)$ denotes the MM constrained optimization procedure described above. The objective function is then calculated as:

$$
L(\theta)=\frac{1}{d_{E}{ }^{2}} \frac{\sum_{i \in N \text { (gridpoints) }} w\left(E_{\mathrm{QM}}\left(\mathbf{x}_{i}\right)\right)\left(E_{\mathrm{QM}}\left(\mathbf{x}_{i}\right)-E_{\mathrm{MM}}\left(\mathbf{x}_{i} ; \theta\right)\right)^{2}}{\sum_{i \in N \text { (gridpoints) }} w\left(E_{\mathrm{QM}}\left(\mathbf{x}_{i}\right)\right)}
$$

where $d_{E}=1.0 \mathrm{kcal} \mathrm{mol}^{-1}$ is a scaling factor. The weights are calculated by a formula that uses two cutoffs, where the weight is constant until the first cutoff $(1.0 \mathrm{kcal} / \mathrm{mol})$ then starts to decrease, followed by a hard second cutoff at $5.0 \mathrm{kcal} / \mathrm{mol}$ above which the weights are zero:

$$
w(E)=\left\{\begin{array}{cc}
1 & E<1.0 \mathrm{kcal} / \mathrm{mol} \\
\sqrt{1+(E-1)^{2}} & 1.0 \leq E<5.0 \mathrm{kcal} / \mathrm{mol} \\
0 & E \geq 5.0 \mathrm{kcal} / \mathrm{mol}
\end{array}\right.
$$

\subsubsection{Optimization algorithm and convergence criteria}

The parameter optimization was carried out with ForceBalance[38], a Python toolkit to optimize force fields in a systematic, reproducible, scalable and flexible manner.[38,58] We employed a development version of ForceBalance based on v1.6.0[59]) to minimize the objective function. Support of the OpenFF force field was enabled by an interface with the OpenFF Toolkit v0.4.1[55]. The commercial OpenEye toolkit version 2019.4.2 was used to generate .mol2 files, which are needed by ForceBalance to set up OpenFF simulations using the toolkit.

Numerical derivatives of the objective function with respect to parameters were computed with dimensionless displacements of 0.01 for improved numerical stability, relative to the ForceBalance default of 0.001 . Fitting was terminated once two convergence criteria were met:

1. The dimensionless parameter step size fell below 0.01 ;

2. The objective function (Section 2.1.7) decreased by less than 0.1 during the step.

To efficiently optimize the parameters in as few iterations as possible, ForceBalance uses a quasi-Newton iteration to take near-optimal steps in parameter space:

$$
\theta^{(n+1)}=\theta^{(n)}+[\mathbf{H}(\theta)+\lambda \mathbf{I}]^{-1}
$$

To approximate the Hessian $\mathbf{H}(\theta)$, ForceBalance computes an approximation to the the matrix of second derivatives of each least-squares component in a manner that neglects parameter couplings:

$$
H_{i, p q}(\theta)=\frac{\partial^{2}}{\partial \theta_{p} \partial \theta_{q}} L_{i}(\theta)=\sum_{j \in \text { data }} \frac{\partial A_{j}}{\partial \theta_{p}} \frac{\partial A_{j}}{\partial \theta_{q}}+\frac{\partial^{2} A_{j}}{\partial \theta_{p} \partial \theta_{q}} \approx \sum_{j \in \text { data }} \frac{\partial A_{j}}{\partial \theta_{p}} \frac{\partial A_{j}}{\partial \theta_{q}}
$$


The $\lambda$ parameter is used to set the optimization step size, and was determined by line-search minimization for a given parametric gradient and Hessian. This strategy was employed because the line search over $\lambda$ only requires repeated evaluation the objective function itself, and not the parametric gradient which is relatively expensive.

\subsubsection{Objective function with regularization}

ForceBalance was used to minimize an objective, or loss function, $L(\theta)$, with respect to force field parameters $\theta$. The objective function quantifies deviation of quantities derived with the force field from the reference quantum chemical data while adding a regularization penalty to minimize the deviation from a reference set of parameters, following the standard approach for ForceBalance[38]:

$$
L_{\mathrm{tot}}(\theta)=\sum_{i \in \text { targets }} w_{i} L_{i}(\theta)+w_{\text {reg }} \sum_{p \in \text { parameters }} \frac{\left|\Delta \theta_{p}\right|^{2}}{\sigma_{p}^{2}}
$$

Here, $w_{i}$ is the weight of each class of optimization data targets with corresponding loss functions $L_{i}(\theta)$, which are often least-squares penalized loss:

$$
L_{i}(\theta)=\sum_{j \in \text { data }}\left(A_{j}^{\text {obs }}-A_{j}^{\text {calc }}(\theta)\right)^{2}
$$

where $A_{j}^{\text {obs }}$ is an observed quantum chemical or physical property target to fit, and $A_{j}^{\text {calc }}(\theta)$ is the calculated value. $w_{i}$ of each target type was chosen to prevent the optimizer prioritizing one target type over other types. Carefully selected weights enabled the objective function contributions of different target types to have the same order of magnitude. $w_{\text {reg }}$ is the regularization penalty weight, and $\Delta \theta$ quantifies the deviation from a reference set of parameters - here, the initial SMIRNOFF99Frosst v 1.1.0 parameter set[33]. Regularization ensures that parameter adjustments are made conservatively to avoid introducing large problematic parameter changes that may only provide marginal improvements in the optimization target, especially when smaller datasets are used in parameterization. We used the regularization scales, $\sigma_{p}$, listed in Table 2, based on past observations of variations in these parameter types in previous studies.[58]

\begin{tabular}{|c|c|}
\hline parameter & regularization scale $\sigma_{p}$ \\
\hline \hline bond force constant $K_{r}$ & $100 \mathrm{kcal} / \mathrm{mol} / \mathrm{A}^{2}$ \\
\hline bond equilibrium length $r_{0}$ & $0.1 \AA$ \\
\hline angle force constant $K_{\theta}$ & $100 \mathrm{kcal} \mathrm{mol}{ }^{-1} \mathrm{rad}^{2}$ \\
\hline angle equilibrium angle $\theta_{0}$ & $20 \mathrm{degrees}$ \\
\hline proper torsion barrier height $K$ & $1 \mathrm{kcal} / \mathrm{mol}$ \\
\hline $\mathrm{vdW}$ well depth $\epsilon$ & $0.1 \mathrm{kcal} / \mathrm{mol}$ \\
\hline $\mathrm{vdW}$ minimum $r_{\text {min-half }}$ & $1 \AA$ \\
\hline
\end{tabular}

Table 2. Regularization scaling parameters used in ForceBalance optimization runs for each force field parameter type.

\subsection{Testing the Parsley Force Field}

Once the parameters had been trained as detailed in Section 2.1, we tested the resulting force field, Parsley1.0.0, against optimized geometries outside the training set, and compared the results to those obtained with the initial force field, SMIRNOFF99Frosst-v1.1.0.[33] We also tested Parsley against two data types outside the training set: energy differences among conformers of a given molecule, and physical properties of various organic liquids. Tests against vibrational spectra and torsional energy potentials are reserved for future studies. Benchmark comparisons of Parsley in the context of other general force fields are also available.[60] We now describe how these tests were done.

\subsubsection{Quantum chemical test set generation}

The QCArchive tool was used to generate and archive additional QM data, using the procedures detailed in Section 2.1, for compounds in three collections. 
OpenFF Discrepancy Benchmark 1 This comprises 2,802 fragment-like molecules (19,712 conformers) selected from the eMolecules catalog[40] because their energy-minimized geometries are substantially different in SMIRNOFF99Frosst 1.0.8 relative to GAFF, GAFF2, MMFF94, and MMFF94s [61, 62]. We retained all protonation and tautomer states present in our initial dataset, but did not generate any additional ones. Further details can be found in Supporting Information section 1.2.1.

Pfizer Discrepancy Optimization Dataset 1 This comprises 100 fragment-like molecules for which Pfizer's QM calculations of torsional profiles computed with HF/minix followed by B3LYP/6-31G*//B3LYP/6$31 \mathrm{G}^{* *}$ differed substantially from those generated with the OPLS3e force field. Pfizer code for relevant related work is public on GitHub.[63] Enumeration of conformers, but not of protonation states, led to 352 conformers.

FDA Optimization Dataset 1 This is a subset of the list of FDA-approved drugs in the ZINC15 FDA dataset[64]. Molecules were kept if they had 3-55 heavy atoms and consisted only of elements $\mathrm{H}, \mathrm{C}, \mathrm{N}, \mathrm{O}, \mathrm{F}, \mathrm{P}, \mathrm{S}, \mathrm{Cl}$, $\mathrm{Br}, \mathrm{I}$ and $\mathrm{B}$. We retained multiple protonation and tautomer states in the database, but did not generate any additional ones. Generation of up to 20 conformers per molecule led to 6,675 conformers for the 1,038 structures.

Test results are presented for the merger of these three datasets, termed the Full Benchmark Set. This dataset can be retrieved from the MQCAS as OpenFF Full Optimization Benchmark 1, as documented elsewhere.[65] This is an "optimization dataset" in the sense that it - and the results presented here for benchmarking on this set - are for performance on optimized geometries only.

Conformational energy differences were assessed as follows. Compound conformers were energyminimized using QM. For a compound with at least three conformers, we identified the conformer $i_{\min }$ with the lowest QM energy $E_{i_{\text {min }, Q M}}$ and computed the energies of its other conformers relative to it: $E_{i, Q M}-$ $E_{i_{\text {min }}, Q M}$. We then computed the force field energies of the same conformers, $E_{i, F F}$, and, for each compound, computed the RMSE of $E_{i, F F}-E_{i_{\min }, F F}$ from the corresponding QM energies. Note that conformation $i_{\min }$ is based on the QM energies and used again for the FF energies.

\subsubsection{Testing against physical properties of organic liquids}

We assessed the ability of molecular dynamics simulations using the newly fitted Parsley force field to replicate 221 experimental observables for organic liquids spanning 104 molecules. The observables used are densities, heats of vaporization, and static dielectric constants, of pure liquids, and excess molar volumes and heats of mixing of binary liquid mixtures. The experimental data were drawn from the NIST ThermoML Archive[66]. For systems involving water, the TIP3P model [67] was used. Automated scripts used to select the data can be found in Supporting Information section 1.2.2.

We started with all available measurements of the properties listed above. When multiple values were available for a given quantity, only the ones with lowest reported uncertainties were retained. We furthermore excluded ionic liquids, compounds containing elements other than $\mathrm{H}, \mathrm{N}, \mathrm{C}, \mathrm{O}, \mathrm{S}, \mathrm{F}, \mathrm{Cl}, \mathrm{Br}$, and I, and data measured outside the temperature range $288-318 \mathrm{~K}$ and the pressure range $0.95-1.05 \mathrm{~atm}$. Dielectric constants $<10$ were also excluded, because a force field that does not explicitly include electronic polarizability is not expected to replicate lower dielectric constants well. The scripts then used a greedy search to identify compounds for which the most data were available and that exercised the largest number of nonbonded parameter types with the fewest compounds. They also weighted smaller compounds more heavily. Sample compounds from the resulting set are shown in Figure 6, and further information on the data set can be found in Supporting Information section1.2.2.

Values for all of these properties were computed with the OpenFF-Evaluator (formerly named the PropertyEstimator) 0.0 .5 tool, using scripts which can be found in Supporting Information section 1.2.2. Calculations were carried out with the new Parsley 1.0.0, and, for comparison, with its precursor, SMIRNOFF99frosst 1.1.0, as well as GAFF 1.81 and GAFF 2.11. 


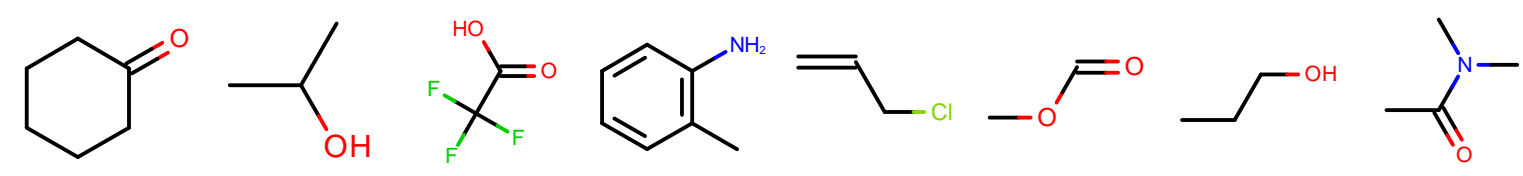

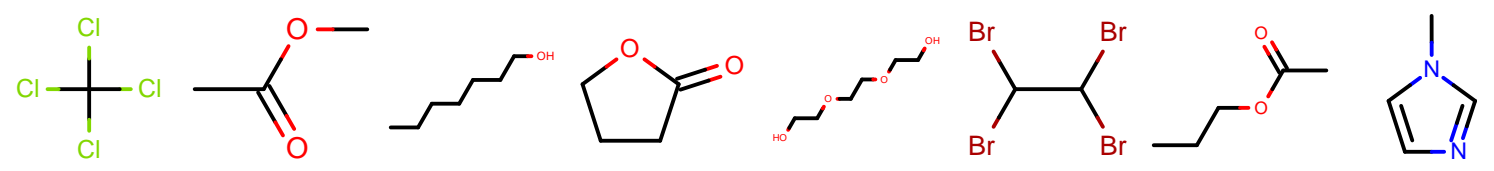<smiles>CCCCOC(C)=O</smiles><smiles>CCCCOC(=O)OCCCO</smiles><smiles>C=C(C)C(=O)OCCO</smiles><smiles></smiles><smiles>CCCC(=O)OC</smiles><smiles>CCCCCCCO</smiles>

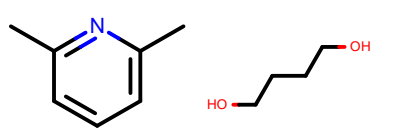

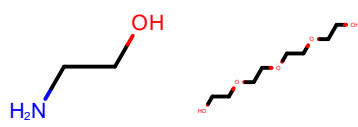
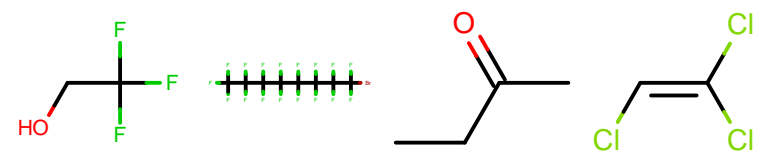<smiles>Oc1ccnc(O)n1</smiles>

Figure 6. Representative molecules in the condensed phase physical property benchmark set.

\section{Results and Discussion}

This section first describes the consequences of parameter optimization for accuracy over the training set, and then benchmarks Parsley on the separate test set compounds and properties. The test set results should be indicative of Parsley's accuracy in new applications. 


\subsubsection{Optimization of the objective function}

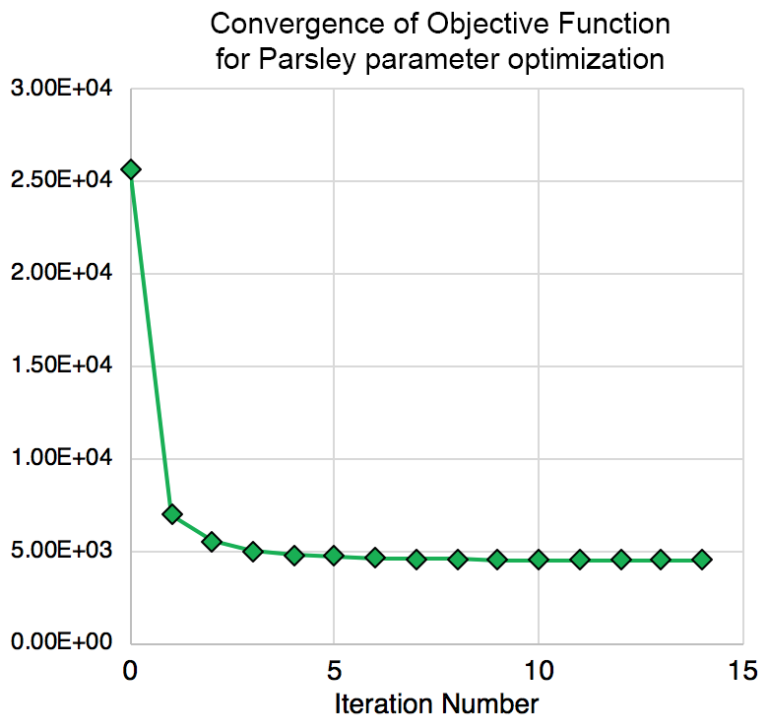

Figure 7. Objective, or loss, function, as a function of number of ForceBalance iterations.

3.1 Improvements in accuracy over training set data

The parameter fitting process dramatically increased the accuracy of the force field for the training data. Although this was anticipated, it is important to confirm, because it verifies the effectiveness of the optimization procedures and provides a scale for the degree of improvement. The dimensionless objective (or loss) function - the weighted sum of squared differences between QM and MM values-decreased dramatically in the fitting, from 25,708 to 4,522 (Figure 7). As described in Section 2.1, the objective function is a sum of contributions which report the accuracy of optimized geometries, vibrational spectra, and torsional energy profiles. The effect of training on these components is summarized in Table 3 (Training Set data) and Figure 8 , and the following subsections provide further details of these results. Full fitting details, as well as inputs and outputs, can be found in the release package[68]. 


\begin{tabular}{|c|c|c|c|c|c|c|c|}
\hline \multirow{2}{*}{\multicolumn{2}{|c|}{ Data class }} & \multicolumn{3}{|c|}{ Training set } & \multicolumn{3}{|c|}{ Full test set } \\
\hline & & $\begin{array}{l}\text { Initial } \\
\text { RMSE }\end{array}$ & $\begin{array}{l}\text { Final } \\
\text { RMSE }\end{array}$ & Change (\%) & $\begin{array}{l}\text { Initial } \\
\text { RMSE }\end{array}$ & $\begin{array}{l}\text { Final } \\
\text { RMSE }\end{array}$ & Change (\%) \\
\hline \multirow{3}{*}{$\begin{array}{l}\text { Geometry } \\
\text { optimization }\end{array}$} & $\begin{array}{l}\text { Bond lengths } \\
\text { (A) }\end{array}$ & 0.045 & 0.023 & $-49 \%$ & 0.023 & 0.017 & $-33 \%$ \\
\hline & $\begin{array}{l}\text { Bond angles } \\
\text { (deg) }\end{array}$ & 3.71 & 3.20 & $-14 \%$ & 3.80 & 3.59 & $-5.5 \%$ \\
\hline & $\begin{array}{l}\text { Improper } \\
\text { dihedrals } \\
\text { (deg) }\end{array}$ & 4.15 & 2.87 & $-31 \%$ & 4.31 & 2.82 & $-35 \%$ \\
\hline $\begin{array}{l}\text { Vibrational } \\
\text { spectra }\end{array}$ & $\begin{array}{l}\text { Frequencies } \\
\left.\qquad \underline{\underline{\left(\mathrm{cm}^{-1}\right.}}\right)\end{array}$ & 119. & 39.6 & $-67 \%$ & ND & ND & ND \\
\hline $\begin{array}{l}\text { Torsion energy } \\
\text { profiles }\end{array}$ & $\begin{array}{l}\text { Energies } \\
\text { (kcal/mol) }\end{array}$ & 2.96 & 1.89 & $-36 \%$ & ND & ND & ND \\
\hline $\begin{array}{l}\text { Relative } \\
\text { energies }\end{array}$ & $\begin{array}{l}\text { Energies } \\
\text { (kcal/mol) }\end{array}$ & ND & ND & ND & 2.43 & 2.13 & $-12 \%$ \\
\hline
\end{tabular}

Table 3. Overall change in root-mean-squared error (RMSE) metrics vs. the quantum chemical result calculated for four types of properties, using the initial and optimized force field, and divided into training set and test set. ND = No Data. 
(a) Optimized geometries, training set

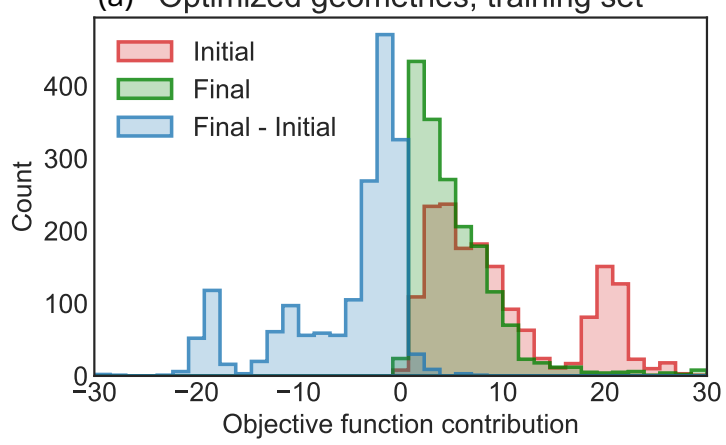

(b) Vibrational spectra, training set

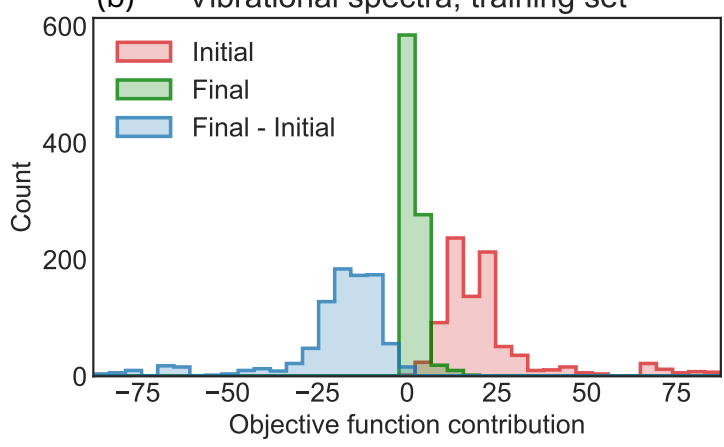

(c) Torsion energy profiles, training set

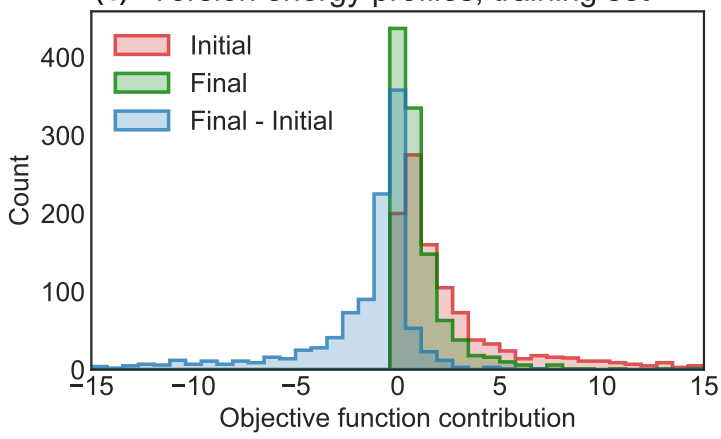

(d) Optimized geometries, full test set

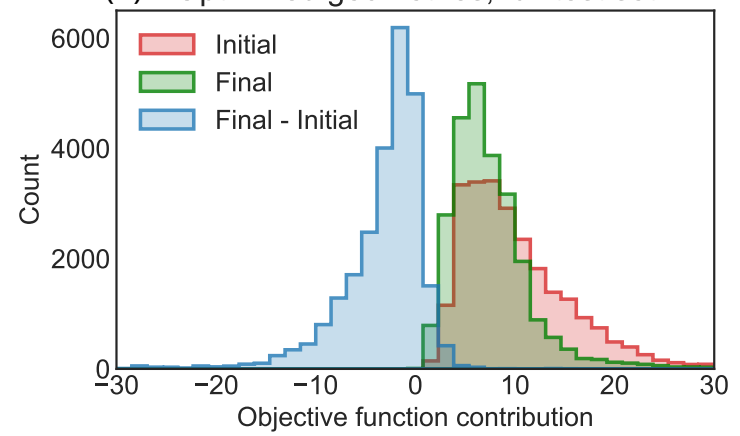

(e) Relative energies, full test set

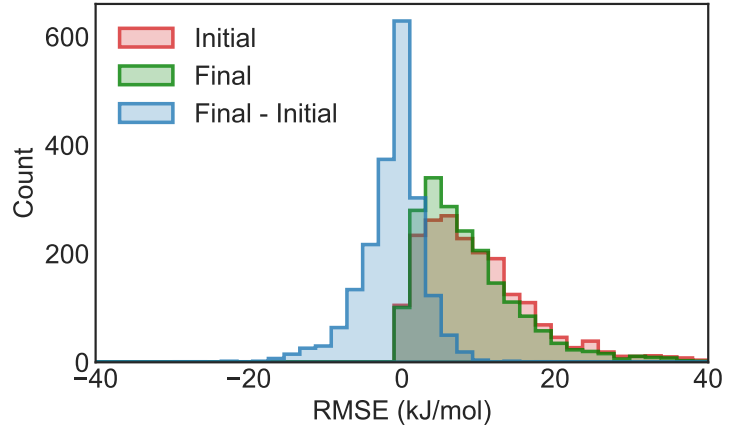

Figure 8. Improvement in components of the training set and test set objective functions with fitting. Red histogram shows performance with our initial force field, green histogram shows performance with the optimized force field and blue histogram shows the distribution of changes in objective function contribution of each target (individual molecules/geometries contributing to the objective function) due to the parameter optimization. Left column (a-c) provides the training set results. Right column (d-e) provides test set results. The range of each plot encompasses $\geq 94.94$ $\%$ of the population of initial objective function contributions and $\geq 99.2 \%$ of the population of final objective function contributions.

\subsubsection{Optimized geometries}

The geometric component of the objective function is computed from the deviations of bond-lengths, bondangles and improper torsions, in structures optimized with the force field, from their values in corresponding structures optimized with QM (Section 2.1.4). As shown in Figure 8a, the fitting process led to improved overall agreement between force field and QM geometries; compare the initial (red) and final (green) histograms. The portion of the blue histogram on the negative / positive $\mathrm{x}$-axis shows the percentage of targets where accuracy is improved / degraded, respectively. The accuracy was somewhat reduced for a small minority of conformers, as evident from the histogram of differences (blue), but this is as expected, because compromises have to be made for some molecules in order to improve the accuracy for others that use the 
same parameters. Table 3 provides a physically interpretable perspective of these results, showing that the RMS errors of bond-lengths, bond-angles, and improper torsions, in the optimized geometries, decreased by $14-49 \%$ with training.

It can also be useful to assess the fitting of individual parameters. To do this for a given bond-stretch parameter, for example, we collected all test cases that included the parameter of interest and made a scatter plot of the length of the bond in the QM geometries vs the length in the MM geometries. Such plots were generated for each bond-length, bond-angle, and improper dihedral, in the training set, and all are available in the release package.[68] When considering this term by term analysis, it should be kept in mind that the length of a bond or the value of an angle in an optimized geometry is determined not just by the parameters of the corresponding force field term, but also by the rest of the structure. For example, a bond length may be shifted by ring strain. However, when these values consistently differ between QM and MM geometries, this can be an indicator that the specific force field parameter requires further attention.

Bond type [\#16X2:1]-[\#8X2:2], N=696

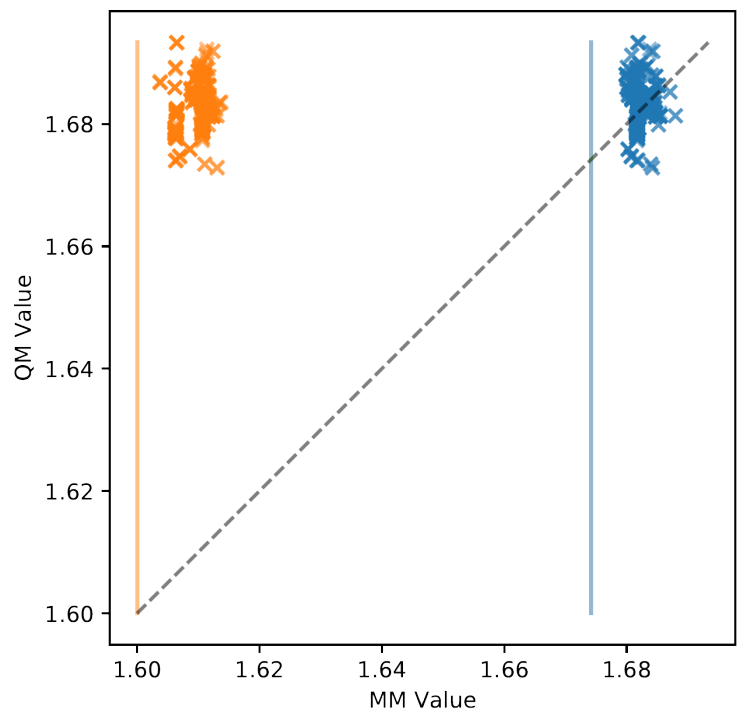

Bond type [\#7:1]-[\#7:2], N=32

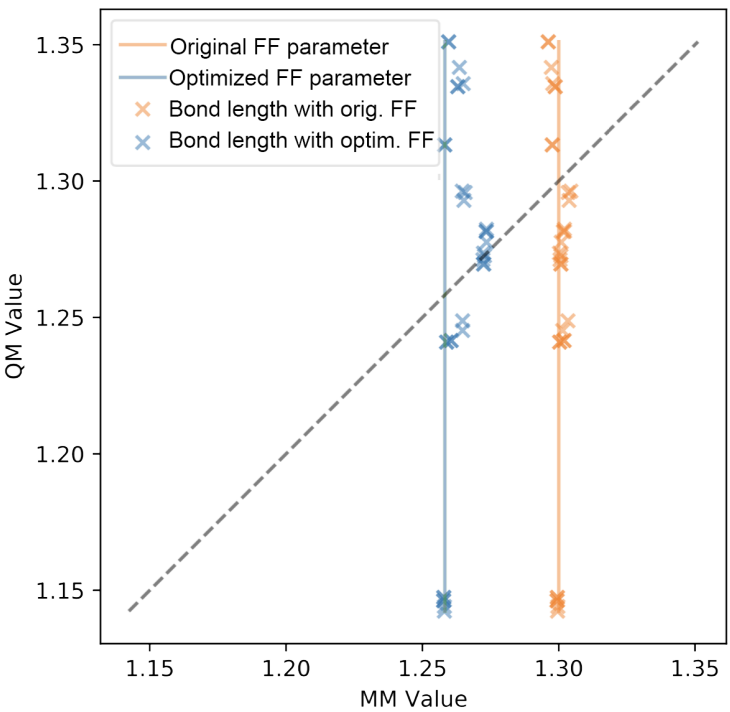

Figure 9. Comparison of QM and MM energy-minimized bond lengths for two parameters. Left: divalent sulfur single-bonded to a divalent oxygen. Right: divalent bond between nitrogens. Vertical line indicates the value of the force field's equilibrium bond length. Orange and blue indicate results for initial and optimized force field. Dashed: Line of identity.

The fitting process moved most bond lengths and angles closer to the diagonals of these scatter plots, implying better agreement between $\mathrm{MM}$ and QM, as expected based on Figure 7. For a clear example, see Figure 9(left), where a change in the equilibrium bond length shifted the MM results to the diagonal and thus into better agreement with QM. However, a few of these scatter plots are more problematic. For example, Figure 9 (right), which examines a general $\mathrm{N}=\mathrm{N}$ bond stretch, shows a small shift of the data points toward the diagonal, but does not correct the fact that this bond length falls in a narrow range across all the MM geometries but is varied in the QM geometries. In cases like this, greater accuracy might be achieved by creating two or more force field types for $\mathrm{N}=\mathrm{N}$ bonds, rather than just one. Before taking such a step, though, one should consider whether the variations in the QM bond lengths trace to varied amounts of strain placed on the bond by other components of the structure. If so, then the accuracy of the $\mathrm{N}=\mathrm{N}$ bond lengths should be improved by adjustment of other parameters that would correct the strain, rather than correcting parameters intrinsic to the bond itself.

Relationships among force field equilibrium bond lengths, chemical environment, and strain, may be further explored by examining the lengths of a given bond type across the geometrically optimized conformers of various compounds. Figure 10 illustrates this concept for a generic C-N single bond. The curves 
in the left panel show the energy-minimized central bond lengths in QM torsion profiles taken from the Roche dataset, including all cases where the central bond is matched by the SMIRKS pattern indicated. The rise and fall of an individual curve indicates a dependence of the central bond length on the torsion angle, and the vertical displacements of the various curves relative to each other indicate the torsion angleindependent differences of central bond lengths between different molecules, or different central bonds in the same molecule. When integrated over all torsion angles, the bond lengths across all instances of the central bond matched by this SMIRKS pattern has a bi- or tri-modal distribution (Figure 10 right panel). This result suggests that this generic bond type ought to be split into at least two or perhaps three more specific types determined by SMIRKS patterns matching more specific chemical environments.

Parameter b7 [\#6:1]-[\#7:2]

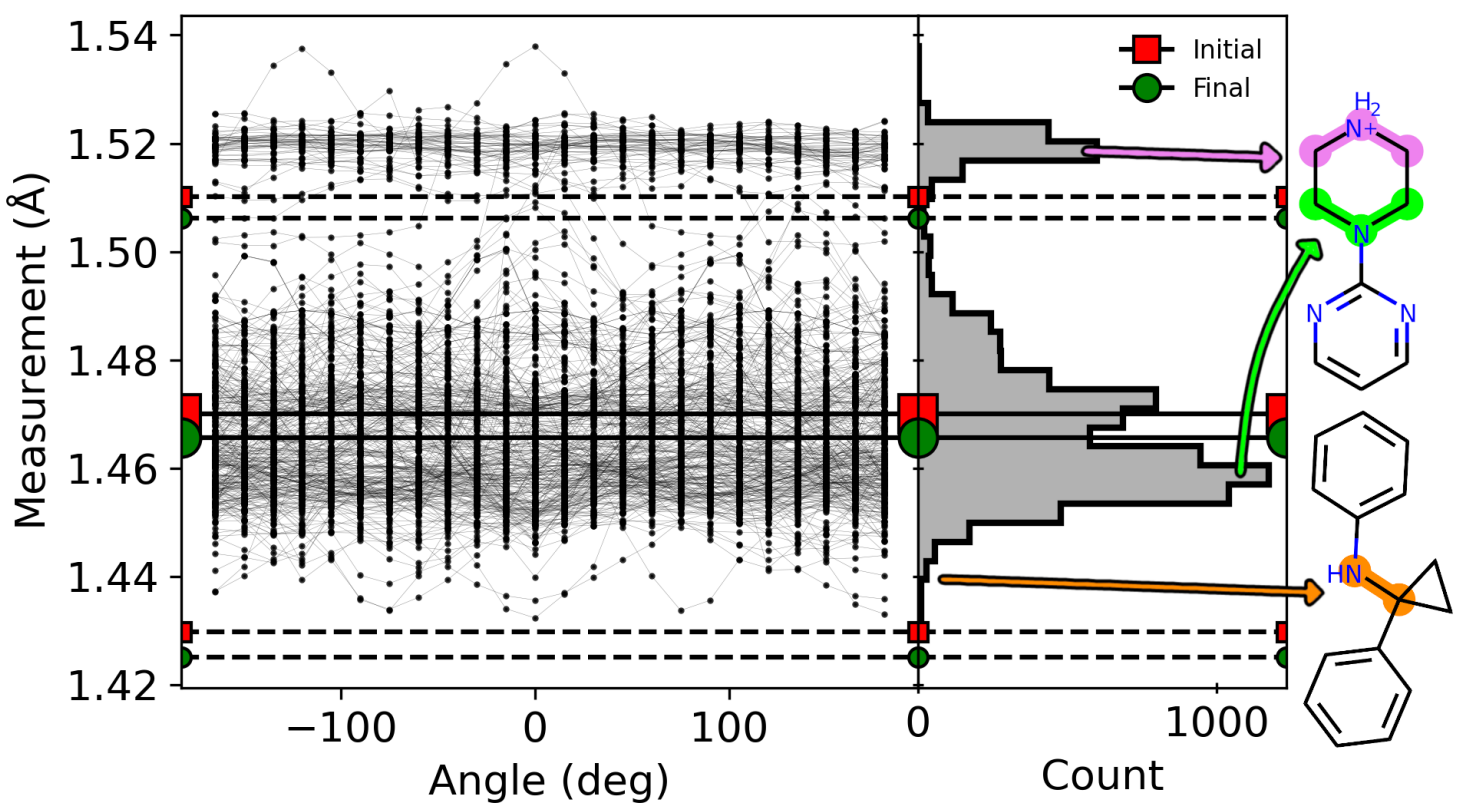

Figure 10. Dependence of bond lengths of a given force field type upon the chemical and conformational environment. Left: Length of the central bond as a function of the torsion angle in the Roche dataset for central bonds matching the $\mathrm{C}-\mathrm{N}$ bond type indicated. Each line corresponds to the length of the $\mathrm{C}-\mathrm{N}$ bond matching the b7 parameter for constrained energy-minimized conformers over a range of torsion angles. Right: Histogram of the observed bond lengths after summing over the torsion angles. Solid line in both panels indicates the force field's equilibrium bond length for type b7, and dashed lines indicate the lengths for which the bond energy equals $1.2 \mathrm{kcal} / \mathrm{mol}$. The lines labeled in red are b7 in the initial force field, and lines labeled in green are b7 after force field optimization. Example molecules and their given b7 bond(s) are highlighted on the far right, which correspond to typical environments where the bond length is $1.44 \AA$ (orange), $1.46 \AA$ (green), or $1.52 \AA$ (violet).

\subsubsection{Vibrational frequencies}

Fitting against the training set led to substantial improvements in the accuracy of the vibrational frequencies in the training data, relative to the reference QM results, as evident from a dramatic fall in the vibrational components of the objective function. This is evident in Figure $8 \mathrm{~b}$, which shows decreases in error for the sum of squared vibrational frequency differences for individual molecules. Indeed, the improvement from initial results (red) to fitted results (green) appears even more marked than that of optimized geometries (Figure 8a). The distribution of improvements for individual conformations (blue) also shows strong improvement, with only a tiny minority of cases becoming less accurate with fitting. These results correspond to a $67 \%$ drop in the RMSE of individual MM vs QM frequencies; i.e., from 119 to $40 \mathrm{~cm}^{-1}$ (Table 3). 


\subsubsection{Torsional energy profiles}

Fitting also led to improvements in the accuracy of the torsional energy profiles in the training set (Figure 8c), although the improvements (red to green) appear less dramatic than for the geometric and vibrational components discussed above. As for the other objective function components, the improvements observed for many torsions come at the expense of decreased accuracy for some others (blue). The RMSE of the MM torsional energy profiles relative to the QM ones in the training set fell from 2.96 to $1.89 \mathrm{kcal} / \mathrm{mol}$, a 36\% drop (Table 3).

It is also of interest to compare the MM and QM potential energy profiles for individual torsion angles across the full training training set, and a full set of comparisons is available in the Supporting Information. Sample plots for a torsional profile that improves with fitting and another that gets worse are provided in Figure 11, left and right panels respectively. Interestingly, the parameter in the second plot occurs 231 times in the training set, so degraded performance is likely not due to lack of sufficient data, but instead to either changes in other portions of the force field, or improved performance on other molecules utilizing this same parameter at the expense of degraded performance for this particular target. Note that most torsional parameters appear in in many molecules in the training set, so fitting can improve accuracy for most occurrences while degrading it for others.

The greater difficulty of fitting torsional profiles may result from the fact that these are particularly sensitive to nonlocal interactions within the molecules, such as longer-range sterics and electrostatics. Also, defining force field types for torsional terms is more complex than for most other terms in the force field, as multiple torsional terms contribute to the profile around a given bond, and torsional terms include step changes in periodicity. Note, too, that the present fitting process adjusted only amplitudes, and left periodicities and phases unchanged. Adjustment of these additional parameters will clearly be of interest in future rounds of force field development.
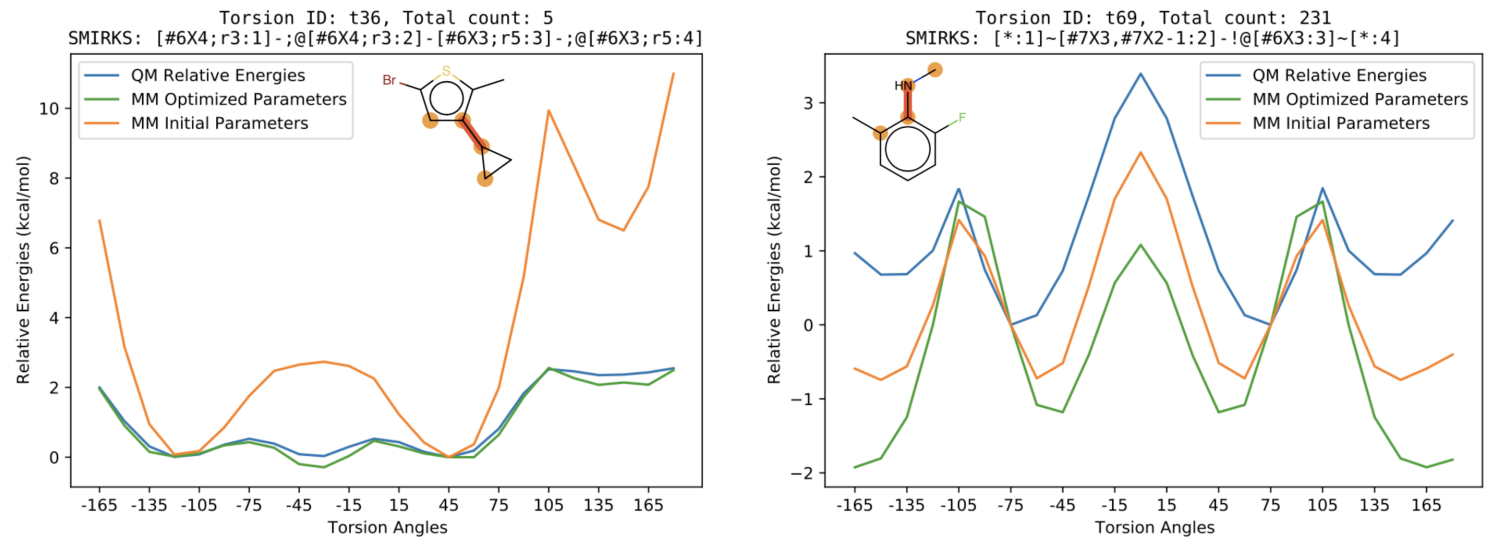

Figure 11. Examples of torsional profiles that were improved (left) or degraded (right) by fitting. Data are for a specific torsion angle in a specific molecule, as detailed below the plot. Blue: QM energy. Orange: force field energy before training. Green: force field energy after training (Parsley). The metadata at the bottom explains which dataset this data is drawn from, and which specific molecule this torsion occurs in, as well as the SMIRKS pattern for the particular torsion being fitted here. The total count of this SMIRKS pattern across the dataset (5) is also shown at the bottom, as well as the parameter ID and the atom indices in the molecule. The full set of plots are available in the release package[68].

\subsection{Test Set Results}

Results for data outside the training set provide an indication of the transferability of the new parameters and hence of the accuracy that may be expected in actual use. Here, we examine the ability of the new parameters to replicate QM-optimized gas-phase geometries for molecules outside training set, energy differences between gas-phase conformers, and, through MD simulations, physical properties of liquids. 


\subsubsection{Quantum chemical data}

The overall objective function for the test set is lower for Parsley $(20,672)$ than for the initial force field $(29,469)$. The distribution of improvements over the test-set compounds (Figure $8 d$ ) shows that the objective function improves for almost all compounds, given that the blue histogram of differences has few positive values. Accordingly, improvements of $6-35 \%$ are observed in the terms that contribute to the objective function (Table 3. It is worth noting that the test set exercises 415 out of the 500 parameters. We also grouped the bond lengths, bond angles, and improper dihedrals across test set compound according to their FF types and examined the improvement in accuracy by type, as illustrated for the bond-lengths in Figure 12. The full figure can be found in Supporting Information Figure 1. Clearly, optimization over the training set led to improved test-improvement for most parameters. Comparable plots for angles and torsions are available in the release package for this force field[69].

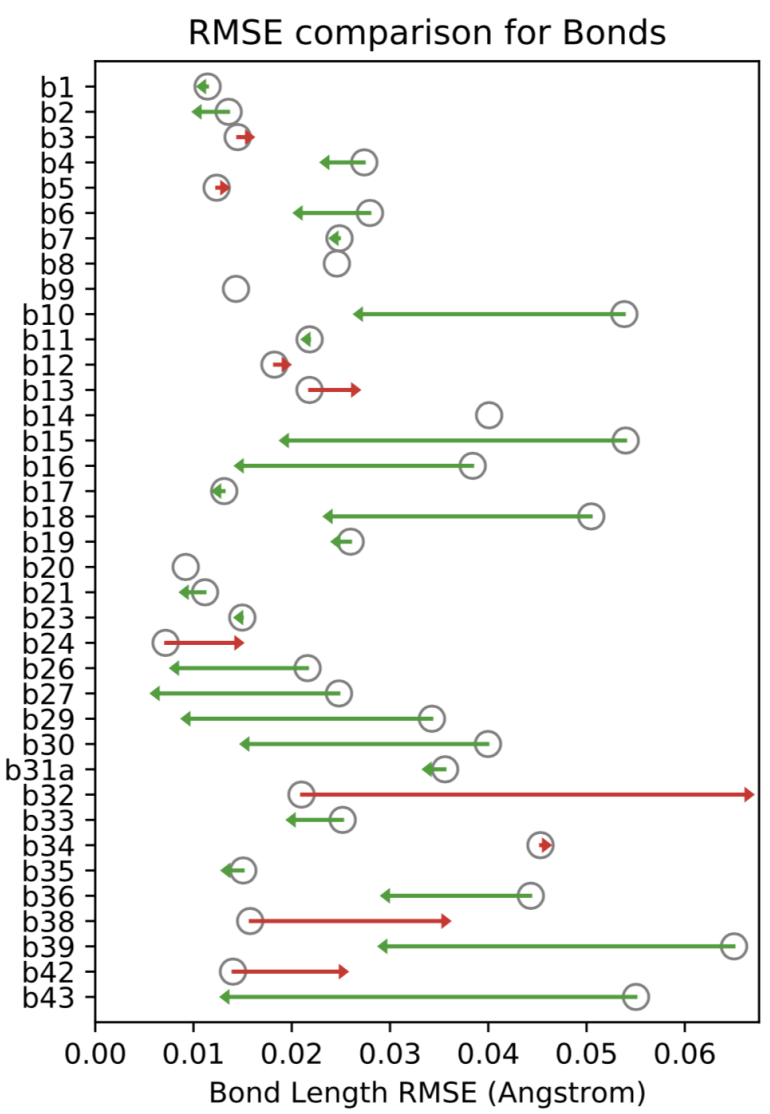

Figure 12. Bond length RMSE comparison for initial and optimized force fields for the Full Set. For each bond type (b1, b2...), a gray circle indicates the RMSE of bonds of this type for the initial force field and arrows show the drops (green) or increases (red) in error on going to the new force field. (SMIRKS patterns for these parameter IDs can be retrieved from the force field XML file, openff-1.0.0-RC2. of $f \operatorname{xml}[39]$.

We also tested the ability of the Parsley force field to replicate differences among conformations of gas-phase molecules in the test set. Note that this type of data is entirely absent from the training data. Nonetheless, the RMSE for these quantities fell by $12 \%$ on going from the initial force field to the new Parsley force field (Table 3). The improvements accuracy are distributed across many compounds, rather than being dominated by improvements for a few, as evident from the histograms in Figure 8e.

\subsubsection{Physical properties of organic liquids}

We tested Parsley's ability to model condensed phase properties by using it to compute densities, enthalpies of vaporization, static dielectric constants, enthalpies of mixing, and excess molar volumes, of organic liq- 
uids and mixtures, and comparing with experimental data from NIST's ThermoML. Note that no condensed phase data were used in the fitting process. As shown in Figures 13 and 14, the new Parsley force field offers competitive performance for these data, with marginal, though not statistically significant (by comparision of the root-mean-square errors and their $95 \%$ confidence intervals), improvement over the previous SMIRNOFF99frosst 1.1.0 release (Table 4). The overall accuracy also is similar to that of the established GAFF family of force fields. This pattern presumably reflects the fact that these physical properties are not sensitive to the valence parameters optimized here, and that condensed phase data were not used to guide the optimization.
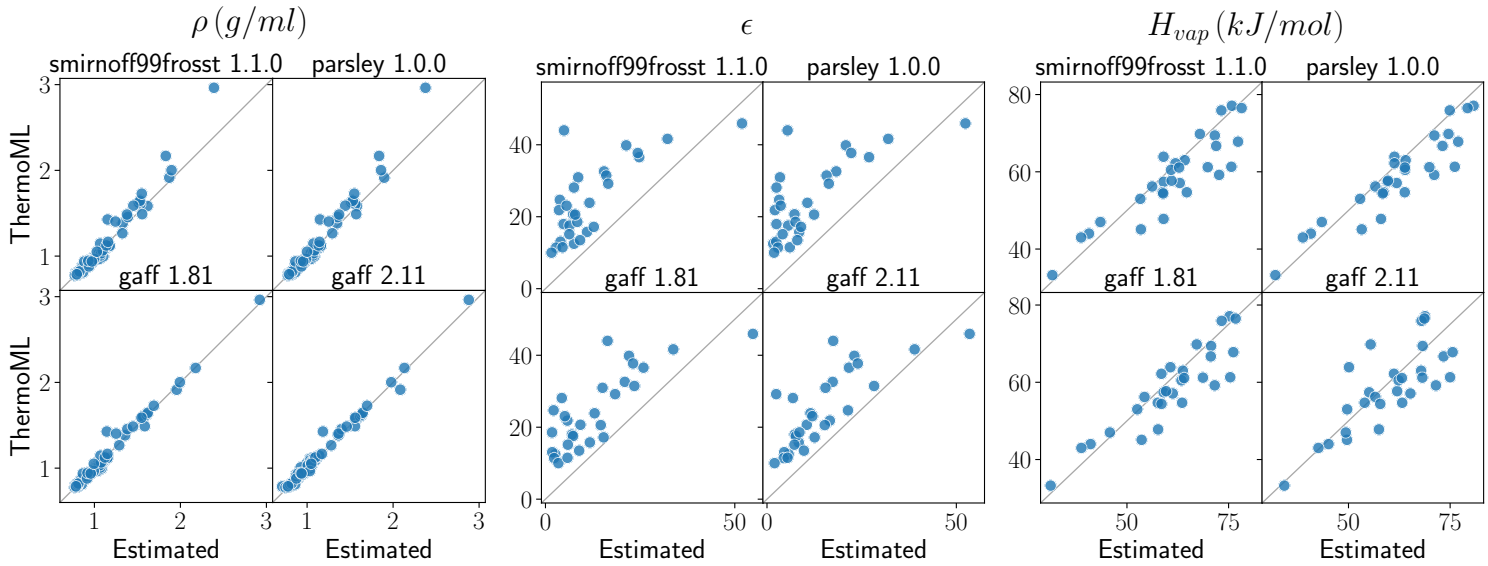

Figure 13. Results of pure property benchmarks. Liquid properties computed with various force fields, as labeled, are compared with experiment. Density: $\rho$; dielectric constant: $\epsilon$; heat of vaporization: $H_{\text {vap }}$;
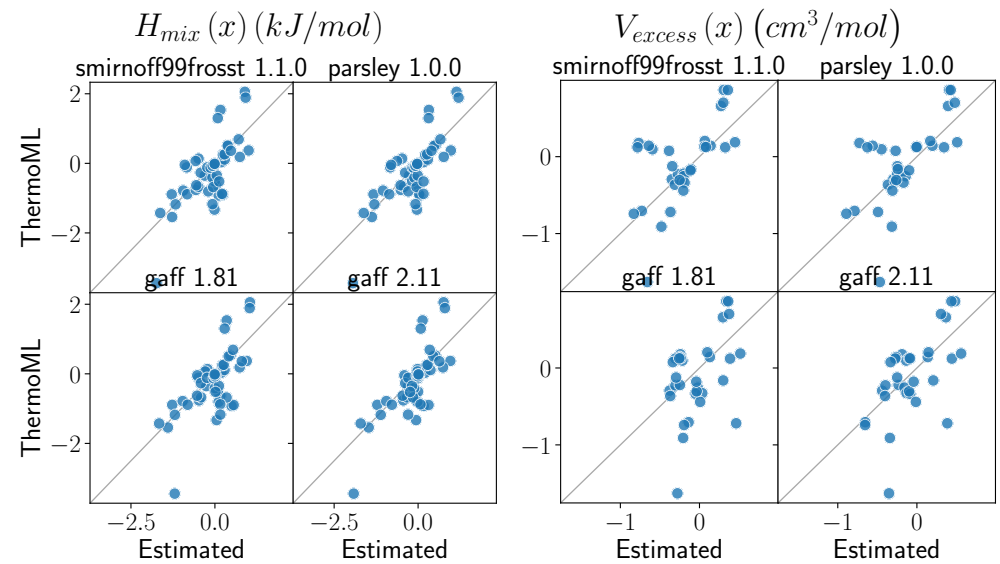

Figure 14. Results of binary mixture property benchmarks. Liquid properties computed with various force fields, as labeled, are compared with experiment. Enthalpy of mixing: $H_{m i x}$; excess molar volume of mixing: $V_{\text {excess }}$. 
Table 4. Measures of accuracy of force fields for the physical property benchmarks. RMSE: root-mean-square error; $R^{2}$ : coefficient of determination; $\tau$ : Kendall's tau ranking accuracy metric. Subscripts and superscripts indicate $95 \%$ confidence intervals on these statistics.

\begin{tabular}{|c|c|c|c|c|}
\hline Property & Force Field & RMSE & $R^{2}$ & $\tau$ \\
\hline \multirow[t]{4}{*}{$V_{\text {excess }}(x)\left(\mathrm{cm}^{3} / \mathrm{mol}\right)$} & smirnoff99frosst 1.1.0 & $0.41_{0.29}^{0.52}$ & $0.39_{0.14}^{0.70}$ & $0.44_{0.13}^{0.67}$ \\
\hline & parsley 1.0 .0 & $0.39_{0.26}^{0.51}$ & $0.44_{0.18}^{0.71}$ & $0.50_{0.22}^{0.71}$ \\
\hline & gaff 1.81 & $0.47_{0.34}^{0.60}$ & $0.17_{0.01}^{0.45}$ & $0.23_{-0.01}^{0.46}$ \\
\hline & gaff 2.11 & $0.41_{0.27}^{0.54}$ & $0.36_{0.10}^{0.66}$ & $0.42_{0.14}^{0.62}$ \\
\hline \multirow{4}{*}{$H_{m i x}(x)(k J / m o l)$} & smirnoff99frosst 1.1.0 & $0.64_{0.50}^{0.71}$ & $0.50_{0.25}^{0.67}$ & $0.49_{0.28}^{0.66}$ \\
\hline & parsley 1.0 .0 & $0.58_{0.46}^{0.69}$ & $0.59_{0.34}^{0.74}$ & $0.54_{0.32}^{0.70}$ \\
\hline & gaff 1.81 & $0.69_{0.52}^{0.80}$ & $0.45_{0.25}^{0.34}$ & $0.44_{0.22}^{0.62}$ \\
\hline & gaff 2.11 & $0.60_{0.45}^{0.72}$ & $0.58_{0.35}^{0.74}$ & $0.58_{0.40}^{0.72}$ \\
\hline \multirow[t]{4}{*}{$H_{\text {vap }}(\mathrm{kJ} / \mathrm{mol})$} & smirnoff99frosst 1.1.0 & $6.03_{4.24}^{7.56}$ & $0.79_{0.62}^{0.90}$ & $0.70_{0.50}^{0.83}$ \\
\hline & parsley 1.0 .0 & $5.87_{4.23}^{7.37}$ & $0.85_{0.71}^{0.93}$ & $0.79_{0.61}^{0.88}$ \\
\hline & gaff 1.81 & $5.53_{3.97}^{7.23}$ & $0.80_{0.63}^{0.91}$ & $0.72_{0.54}^{0.81}$ \\
\hline & gaff 2.11 & $7.00_{5.33}^{8.51}$ & $0.60_{0.30}^{0.80}$ & $0.59_{0.35}^{0.74}$ \\
\hline \multirow[t]{4}{*}{$\rho(g / m l)$} & smirnoff99frosst 1.1.0 & $0.10_{0.06}^{0.14}$ & $0.96_{0.94}^{0.98}$ & $0.90_{0.85}^{0.92}$ \\
\hline & parsley 1.0.0 & $0.10_{0.05}^{0.15}$ & $0.96_{0.94}^{0.98}$ & $0.90_{0.85}^{0.92}$ \\
\hline & gaff 1.81 & $0.05_{0.03}^{0.07}$ & $0.98_{0.95}^{1.00}$ & $0.91_{0.87}^{0.94}$ \\
\hline & gaff 2.11 & $0.05_{0.03}^{0.07}$ & $0.99_{0.97}^{1.00}$ & $0.92_{0.87}^{0.94}$ \\
\hline \multirow[t]{4}{*}{$\epsilon$} & smirnoff99frosst 1.1.0 & $14.78_{11.62}^{18.17}$ & $0.53_{0.22}^{0.80}$ & $0.50_{0.22}^{0.72}$ \\
\hline & parsley 1.0.0 & $15.72_{12.54}^{19.19}$ & $0.53_{0.22}^{0.79}$ & $0.49_{0.20}^{0.69}$ \\
\hline & gaff 1.81 & $13.22_{10.80}^{15.63}$ & $0.64_{0.44}^{0.82}$ & $0.54_{0.34}^{0.73}$ \\
\hline & gaff 2.11 & $12.16_{9.40}^{14.96}$ & $0.62_{0.40}^{0.84}$ & $0.67_{0.42}^{0.34}$ \\
\hline
\end{tabular}

\section{Using and Citing Parsley}

The present Parsley force field, formally named openff-1.0.0, can be accessed from Python by installing the Open Force Field Toolkit with the command conda install -c omnia openforcefield openforcefields and then loading the force field as follows:

from openforcefield.typing.engines.smirnoff import ForceField

$f f=$ ForceField ('openff-1.0.0. offxml')

The default version of Parsley includes hydrogen bond length constraints, which allow use of the typical 2-4 fs timestep in molecular dynamics simulations. A second version without these constraints, which is suitable for geometry optimizations and single-point energy calculations, may be accessed as follows:

$f f=$ ForceField('openff_unconstrained-1.0.0.offxml')

An example of the use of Parsley to run a molecular dynamics simulation can be found in Supporting Information section 3. Alternatively, the force field files themselves can be found under the openforcefields/offxml subdirectory of the openforcefields GitHub repository[70].

The present Parsley version may be referred to as "Open Force Field (OpenFF) Parsley Force Field (v1.0.0)" on first reference, and "Parsley" thereafter. Newer Open Force Fields are in development, and updates in the OpenFF 1.x series will also be referred to as Parsley, while new major versions will receive updated codenames. To cite Parsley, please reference the latest version of this article and the DOI of the force field version you use. This information is available in the OpenForceField repository[70], and the present version may be cited as [39].

To provide feedback on the performance of the OpenFF force fields, we highly recommend using the issue tracker at http://github.com/openforcefield/openforcefields. For toolkit feedback, use http://github.com/ openforcefield/openforcefield. Alternatively, inquiries may be e-mailed to support@openforcefield.org, though responses to e-mails sent to this address may be delayed and GitHub issues receive higher priority. For 
information on getting started with OpenFF, please see the documentation linked at http://github.com/ openforcefield/openforcefield, and note the availability of several introductory examples.

\section{Conclusions and Future Directions}

We have reported a new force field with bonded, or valence, terms optimized against a range of gas-phase QM reference data. The resulting Parsley v.1.0.0 provides more accurate molecular geometries and conformational energetics, while preserving accuracy for a range of condensed phase properties. However, more benchmarking and testing would be of value. Areas of particular interest include:

- Broadened range of chemistries

- Additional condensed-phase properties, such as hydration and transfer free energies

- Host-guest binding free energies

- Protein-ligand binding free energies

- Small molecule conformer generation and optimization

- Ligand strain energy in protein-ligand crystal structures

- Further comparisons with other force fields

Work along these lines has already begun [60], and we look forward to interacting with others in the community to continue assessing the performance of this and future OpenFF force fields.

\section{Acknowledgements}

In this work, we stand on the shoulders of giants in the force field development community, and it would be impossible to provide a complete list of all those whose work we have benefitted from. However, we are particularly indebted to the AMBER force field community, from which we derived the initial small molecule force fields which provided the starting point for this work. We thank the Open Force Field Consortium for funding, including our industry partners as listed at the Open Force Field website[71]. We gratefully acknowledge Owen Madin and Karmen Condic-Jurkic for helpful discussions, along with all current and former members of the Open Force Field Initiative and the Open Force Field Scientific Advisory Board. We thank the National Institutes of Health (NIGMS R01GM132386) for funding longer-term aspects of this initiative. MKG acknowledges funding from National Institute of General Medical Sciences (GM061300). LPW and YQ acknowledge support from ACS PRF 58158-DNI6. MRS and JDC acknowledge support from NSF CHE-1738975. DGAS acknowledges funding from the U. S. National Science Foundation (NSF) grant ACl-1547580. These findings are solely of the authors and do not necessarily represent the views of the NIH or NSF.

\section{Disclosures}

The Chodera laboratory receives or has received funding from multiple sources, including the National Institutes of Health, the National Science Foundation, the Parker Institute for Cancer Immunotherapy, Relay Therapeutics, Entasis Therapeutics, Silicon Therapeutics, EMD Serono (Merck KGaA), AstraZeneca, Vir Biotechnology, XtalPi, Foresite Labs, the Molecular Sciences Software Institute, the Starr Cancer Consortium, the Open Force Field Consortium, Cycle for Survival, a Louis V. Gerstner Young Investigator Award, and the Sloan Kettering Institute. A complete funding history for the Chodera lab can be found at http: //choderalab.org/funding.

JDC is a current member of the Scientific Advisory Board of OpenEye Scientific Software and scientific consultant to Foresite Labs.

MRS is an Open Science Fellow for Silicon Therapeutics.

MKG has an equity interest in and is a cofounder and scientific advisor of VeraChem LLC.

DLM is a current member of the Scientific Advisory Board of OpenEye Scientific Software and an Open Science Fellow for Silicon Therapeutics. 


\section{References}

[1] Coleman RG, Carchia M, Sterling T, Irwin JJ, Shoichet BK. Ligand Pose and Orientational Sampling in Molecular Docking. PLOS ONE. 2013 10; 8(10):1-19. https://doi.org/10.1371/journal.pone.0075992, doi: 10.1371/journal.pone.0075992.

[2] Allen WJ, Balius TE, Mukherjee S, Brozell SR, Moustakas DT, Lang PT, Case DA, Kuntz ID, Rizzo RC. DOCK 6: Impact of new features and current docking performance. Journal of Computational Chemistry. 2015; 36(15):1132-1156. https://onlinelibrary.wiley.com/doi/abs/10.1002/jcc.23905, doi: 10.1002/jcc.23905.

[3] Österberg F, Morris GM, Sanner MF, Olson AJ, Goodsell DS. Automated docking to multiple target structures: Incorporation of protein mobility and structural water heterogeneity in AutoDock. Proteins: Structure, Function, and Bioinformatics. 2002; 46(1):34-40. https://onlinelibrary.wiley.com/doi/abs/10.1002/prot.10028, doi: 10.1002/prot.10028.

[4] Friesner RA, Banks JL, Murphy RB, Halgren TA, Klicic JJ, Mainz DT, Repasky MP, Knoll EH, Shelley M, Perry JK, Shaw DE, Francis P, Shenkin PS. Glide: A New Approach for Rapid, Accurate Docking and Scoring. 1. Method and Assessment of Docking Accuracy. Journal of Medicinal Chemistry. 2004; 47(7):1739-1749. https://doi.org/10.1021/jm0306430, doi: 10.1021/jm0306430, pMID: 15027865.

[5] Jain AN. Surflex: Fully Automatic Flexible Molecular Docking Using a Molecular Similarity-Based Search Engine. Journal of Medicinal Chemistry. 2003; 46(4):499-511. https://doi.org/10.1021/jm020406h, doi: 10.1021/jm020406h, pMID: 12570372.

[6] Jones G, Willett P, Glen RC, Leach AR, Taylor R. Development and validation of a genetic algorithm for flexible docking. Journal of Molecular Biology. 1997; 267(3):727 - 748. http://www.sciencedirect.com/science/article/pii/ S0022283696908979, doi: 10.1006/jmbi.1996.0897.

[7] McGann MR, Almond HR, Nicholls A, Grant JA, Brown FK. Gaussian docking functions. Biopolymers. 2003; 68(1):7690. https://onlinelibrary.wiley.com/doi/abs/10.1002/bip.10207, doi: 10.1002/bip.10207.

[8] Lamb ML, Jorgensen WL. Computational approaches to molecular recognition. Current Opinion in Chemical Biology. 1997; 1(4):449 - 457. http://www.sciencedirect.com/science/article/pii/S1367593197800385, doi: https://doi.org/10.1016/S1367-5931(97)80038-5.

[9] Gilson MK, Zhou HX. Calculation of Protein-Ligand Binding Affinities. Annual Review of Biophysics and Biomolecular Structure. 2007; 36(1):21-42. https://doi.org/10.1146/annurev.biophys.36.040306.132550, doi: 10.1146/annurev.biophys.36.040306.132550, pMID: 17201676.

[10] Gallicchio E, Levy RM. Recent theoretical and computational advances for modeling protein-ligand binding affinities. In: Christov C, editor. Computational chemistry methods in structural biology, vol. 85 of Advances in Protein Chemistry and Structural Biology Academic Press; 2011.p. 27 - 80. http://www.sciencedirect.com/science/article/pii/ B9780123864857000028, doi: https://doi.org/10.1016/B978-0-12-386485-7.00002-8.

[11] Huggins DJ, Biggin PC, Dämgen MA, Essex JW, Harris SA, Henchman RH, Khalid S, Kuzmanic A, Laughton CA, Michel J, Mulholland AJ, Rosta E, Sansom MSP, van der Kamp MW. Biomolecular simulations: From dynamics and mechanisms to computational assays of biological activity. WIREs Computational Molecular Science. 2019; 9(3):e1393. https: //onlinelibrary.wiley.com/doi/abs/10.1002/wcms.1393, doi: 10.1002/wcms.1393.

[12] Wang L, Wu Y, Deng Y, Kim B, Pierce L, Krilov G, Lupyan D, Robinson S, Dahlgren MK, Greenwood J, Romero DL, Masse C, Knight JL, Steinbrecher T, Beuming T, Damm W, Harder E, Sherman W, Brewer M, Wester R, et al. Accurate and Reliable Prediction of Relative Ligand Binding Potency in Prospective Drug Discovery by Way of a Modern FreeEnergy Calculation Protocol and Force Field. Journal of the American Chemical Society. 2015; 137(7):2695-2703. https://doi.org/10.1021/ja512751q, doi: 10.1021/ja512751q, pMID: 25625324.

[13] Cournia Z, Allen B, Sherman W. Relative Binding Free Energy Calculations in Drug Discovery: Recent Advances and Practical Considerations. Journal of Chemical Information and Modeling. 2017; 57(12):2911-2937. https://doi.org/ 10.1021/acs.jcim.7b00564, doi: 10.1021/acs.jcim.7b00564, pMID: 29243483.

[14] Schindler C, Baumann H, Blum A, Böse D, Buchstaller HP, Burgdorf L, Cappel D, Chekler E, Czodrowski P, Dorsch D, Eguida M, Follows B, Fuchß T, Grädler U, Gunera J, Johnson T, Lebrun CJ, Karra S, Klein M, Kötzner L, et al., Large-Scale Assessment of Binding Free Energy Calculations in Active Drug Discovery Projects; 2020. https://chemrxiv.org/articles/preprint/Large-Scale_Assessment_of_Binding_Free_Energy_Calculations_in_ Active Drug Discovery Projects/11364884, doi: 10.26434/chemrxiv. $\overline{1} 13 \overline{6} 4884 . v 2$. 
[15] Cournia Z, Allen BK, Beuming T, Pearlman DA, Radak BK, Sherman W. Rigorous Free Energy Simulations in Virtual Screening. Journal of Chemical Information and Modeling. 2020; 60(9):4153-4169. https://doi.org/10.1021/acs.jcim. 0c00116, doi: 10.1021/acs.jcim.0c00116, pMID: 32539386.

[16] Cornell WD, Cieplak P, Bayly Cl, Gould IR, Merz KM, Ferguson DM, Spellmeyer DC, Fox T, Caldwell JW, Kollman PA. A Second Generation Force Field for the Simulation of Proteins, Nucleic Acids, and Organic Molecules. Journal of the American Chemical Society. 1995; 117(19):5179-5197. https://doi.org/10.1021/ja00124a002, doi: $10.1021 / j a 00124 a 002$.

[17] MacKerell AD, Bashford D, Bellott M, Dunbrack RL, Evanseck JD, Field MJ, Fischer S, Gao J, Guo H, Ha S, JosephMcCarthy D, Kuchnir L, Kuczera K, Lau FTK, Mattos C, Michnick S, Ngo T, Nguyen DT, Prodhom B, Reiher WE, et al. All-Atom Empirical Potential for Molecular Modeling and Dynamics Studies of Proteins. The Journal of Physical Chemistry B. 1998; 102(18):3586-3616. https://doi.org/10.1021/.jp973084f, doi: 10.1021/jp973084f, pMID: 24889800.

[18] Foloppe N, MacKerell AD Jr. All-atom empirical force field for nucleic acids: I. Parameter optimization based on small molecule and condensed phase macromolecular target data. Journal of Computational Chemistry. 2000; 21(2):86104. doi: 10.1002/(SICI)1096-987X(20000130)21:2<86::AID-JCC2>3.0.CO;2-G.

[19] MacKerell Jr AD, Banavali NK. All-atom empirical force field for nucleic acids: II. Application to molecular dynamics simulations of DNA and RNA in solution. Journal of Computational Chemistry. 2000; 21(2):105-120. doi: 10.1002/(SICI)1096-987X(20000130)21:2<105::AID-JCC3>3.0.CO;2-P.

[20] Hornak V, Abel R, Okur A, Strockbine B, Roitberg A, Simmerling C. Comparison of multiple Amber force fields and development of improved protein backbone parameters. Proteins: Structure, Function, and Bioinformatics. 2006; 65(3):712-725. https://onlinelibrary.wiley.com/doi/abs/10.1002/prot.21123, doi: 10.1002/prot.21123.

[21] Lindorff-Larsen K, Piana S, Palmo K, Maragakis P, Klepeis JL, Dror RO, Shaw DE. Improved side-chain torsion potentials for the Amber ff99SB protein force field. Proteins: Structure, Function, and Bioinformatics. 2010; 78(8):19501958. https://onlinelibrary.wiley.com/doi/abs/10.1002/prot.22711, doi: 10.1002/prot.22711.

[22] Wang LP, McKiernan KA, Gomes J, Beauchamp KA, Head-Gordon T, Rice JE, Swope WC, Martínez TJ, Pande VS. Building a More Predictive Protein Force Field: A Systematic and Reproducible Route to AMBER-FB15. The Journal of Physical Chemistry B. 2017; 121(16):4023-4039. https://doi.org/10.1021/acs.jpcb.7b02320, doi: 10.1021/acs.jpcb.7b02320, pMID: 28306259.

[23] Oostenbrink C, Villa A, Mark AE, Van Gunsteren WF. A biomolecular force field based on the free enthalpy of hydration and solvation: The GROMOS force-field parameter sets 53A5 and 53A6. Journal of Computational Chemistry. 2004; 25(13):1656-1676. https://onlinelibrary.wiley.com/doi/abs/10.1002/jcc.20090, doi: 10.1002/jcc.20090.

[24] Shi Y, Xia Z, Zhang J, Best R, Wu C, Ponder JW, Ren P. Polarizable Atomic Multipole-Based AMOEBA Force Field for Proteins. Journal of Chemical Theory and Computation. 2013; 9(9):4046-4063. https://doi.org/10.1021/ct4003702, doi: 10.1021/ct4003702, pMID: 24163642.

[25] Kim S, Thiessen PA, Bolton EE, Chen J, Fu G, Gindulyte A, Han L, He J, He S, Shoemaker BA, Wang J, Yu B, Zhang J, Bryant SH. PubChem Substance and Compound databases. Nucleic Acids Research. 2016 Jan; 44(D1):D1202-D1213. https://academic.oup.com/nar/article/44/D1/D1202/2503131, doi: 10.1093/nar/gkv951, publisher: Oxford Academic.

[26] Wang J, Wolf RM, Caldwell JW, Kollman PA, Case DA. Development and testing of a general amber force field. Journal of Computational Chemistry. 2004 Jul; 25(9):1157-1174. doi: 10.1002/jcc.20035.

[27] Vanommeslaeghe K, Hatcher E, Acharya C, Kundu S, Zhong S, Shim J, Darian E, Guvench O, Lopes P, Vorobyov I, Mackerell AD. CHARMM general force field: A force field for drug-like molecules compatible with the CHARMM all-atom additive biological force fields. Journal of Computational Chemistry. 2010; 31(4). doi: 10.1002/jcc.21367.

[28] Jorgensen WL, Maxwell DS, Tirado-Rives J. Development and Testing of the OPLS All-Atom Force Field on Conformational Energetics and Properties of Organic Liquids. Journal of the American Chemical Society. 1996; 118(45):1122511236. doi: 10.1021/ja9621760.

[29] Fennell CJ, Wymer KL, Mobley DL. A Fixed-Charge Model for Alcohol Polarization in the Condensed Phase, and Its Role in Small Molecule Hydration. The Journal of Physical Chemistry B. 2014; 118(24):6438-6446. https://doi.org/10. 1021/jp411529h, doi: 10.1021/jp411529h, pMID: 24702668.

[30] Henriksen NM, Fenley AT, Gilson MK. Computational Calorimetry: High-Precision Calculation of Host-Guest Binding Thermodynamics. Journal of Chemical Theory and Computation. 2015; 11(9):4377-4394. https://doi.org/10.1021/ acs.jctc.5b00405, doi: 10.1021/acs.jctc.5b00405, pMID: 26523125. 
[31] Yin J, Henriksen NM, Muddana HS, Gilson MK. Bind3P: Optimization of a Water Model Based on Host-Guest Binding Data. Journal of Chemical Theory and Computation. 2018; 14(7):3621-3632. https://doi.org/10.1021/acs.jctc.8b00318, doi: 10.1021/acs.jctc.8b00318, pMID: 29874074.

[32] Kenney IM, Beckstein O, lorga BI. Prediction of cyclohexane-water distribution coefficients for the SAMPL5 data set using molecular dynamics simulations with the OPLS-AA force field. Journal of computer-aided molecular design. 2016 November; 30(11):1045-1058. https://doi.org/10.1007/s10822-016-9949-5, doi: 10.1007/s10822-016-9949-5.

[33] Mobley DL, Bannan CC, Rizzi A, Bayly Cl, Chodera JD, Lim VT, Lim NM, Beauchamp KA, Slochower DR, Shirts MR, Gilson MK, Eastman PK. Escaping Atom Types in Force Fields Using Direct Chemical Perception. Journal of Chemical Theory and Computation. 2018; 14(11):6076-6092. https://doi.org/10.1021/acs.jctc.8b00640, doi: 10.1021/acs.jctc.8b00640, pMID: 30351006.

[34] Bayly C, McKay D, Truchon J, An Informal AMBER Small Molecule Force Field: Parm@ Frosst; 2010. http://www.ccl.net/cca/data/parm_at_Frosst/.

[35] Slochower DR, Henriksen NM, Wang LP, Chodera JD, Mobley DL, Gilson MK. Binding Thermodynamics of Host-Guest Systems with SMIRNOFF99Frosst 1.0.5 from the Open Force Field Initiative. Journal of Chemical Theory and Computation. 2019; 15(11):6225-6242. doi: 10.1021/acs.jctc.9b00748.

[36] Jakalian A, Jack DB, Bayly Cl. Fast, efficient generation of high-quality atomic charges. AM1-BCC model: II. Parameterization and validation. Journal of Computational Chemistry. 2002; 23(16):1623-1641. https://onlinelibrary.wiley. com/doi/abs/10.1002/jcc.10128, doi: 10.1002/jcc.10128.

[37] Wang J, Cieplak P, Kollman PA. How well does a restrained electrostatic potential (RESP) model perform in calculating conformational energies of organic and biological molecules? Journal of Computational Chemistry. 2000; 21(12):1049-1074. doi: 10.1002/1096-987X(200009)21:12<1049::AID-JCC3>3.0.CO;2-F.

[38] Wang LP, Martinez TJ, Pande VS. Building Force Fields: An Automatic, Systematic, and Reproducible Approach. The Journal of Physical Chemistry Letters. 2014; 5(11):1885-1891. https://doi.org/10.1021/jz500737m, doi: 10.1021/jz500737m, pMID: 26273869.

[39] Wagner J, openforcefield/openforcefields: Version 1.0.0 "Parsley". Zenodo; 2019. https://doi.org/10.5281/zenodo. 3483227, doi: 10.5281/zenodo.3483227.

[40] eMolecules Plus Database Download; 2013. https://www.emolecules.com/info/plus/download-database.

[41] MolSSI QCFractal Documentation; 2019. https://qcfractal.readthedocs.io.

[42] MolSSI QCArchive web page; 2020. https://qcarchive.molssi.org/.

[43] Smith DGA, Altarawy D, Burns LA, Welborn M, Naden LN, Ward L, Ellis S, Pritchard BP, Crawford TD. The MolSSI QCArchive project: An open-source platform to compute, organize, and share quantum chemistry data. WIREs Computational Molecular Science. ; n/a(n/a):e1491. https://onlinelibrary.wiley.com/doi/abs/10.1002/wcms.1491, doi: 10.1002/wcms.1491.

[44] Godbout N, Salahub DR, Andzelm J, Wimmer E. Optimization of Gaussian-type basis sets for local spin density functional calculations. Part I. Boron through neon, optimization technique and validation. Canadian Journal of Chemistry. 1992; 70(2):560-571. https://doi.org/10.1139/v92-079, doi: 10.1139/v92-079.

[45] Becke AD. Density-functional thermochemistry. III. The role of exact exchange. The Journal of Chemical Physics. 1993; 98(7):5648-5652. https://doi.org/10.1063/1.464913, doi: 10.1063/1.464913.

[46] Grimme S, Antony J, Ehrlich S, Krieg H. A consistent and accurate ab initio parametrization of density functional dispersion correction (DFT-D) for the 94 elements H-Pu. The Journal of Chemical Physics. 2010; 132(15):154104. https://doi.org/10.1063/1.3382344, doi: 10.1063/1.3382344.

[47] Grimme S, Ehrlich S, Goerigk L. Effect of the damping function in dispersion corrected density functional theory. Journal of Computational Chemistry. 2011; 32(7):1456-1465. https://onlinelibrary.wiley.com/doi/abs/10.1002/jcc. 21759, doi: 10.1002/jcc.21759.

[48] Řezáč J, Bím D, Gutten O, Rulíšek L. Toward Accurate Conformational Energies of Smaller Peptides and MediumSized Macrocycles: MPCONF196 Benchmark Energy Data Set. Journal of Chemical Theory and Computation. 2018; 14(3):1254-1266. https://doi.org/10.1021/acs.jctc.7b01074, doi: 10.1021/acs.jctc.7b01074, pMID: 29461829. 
[49] Kesharwani MK, Karton A, Martin JML. Benchmark ab Initio Conformational Energies for the Proteinogenic Amino Acids through Explicitly Correlated Methods. Assessment of Density Functional Methods. Journal of Chemical Theory and Computation. 2016; 12(1):444-454. https://doi.org/10.1021/acs.jctc.5b01066, doi: 10.1021/acs.jctc.5b01066, pMID: 26653705.

[50] Weigend F, Ahlrichs R. Balanced basis sets of split valence, triple zeta valence and quadruple zeta valence quality for $\mathrm{H}$ to Rn: Design and assessment of accuracy. Phys Chem Chem Phys. 2005; 7:3297-3305. http://dx.doi.org/10. 1039/B508541A, doi: 10.1039/B508541A.

[51] Development version of fragmenter used for 1.0 .0 valence parameter fitting; 2019 . https://github.com/ openforcefield/fragmenter/tree/87b85a406aa9c6ac0cfbaf582ed05c55799161a9.

[52] Wang LP, Song C. Geometry optimization made simple with translation and rotation coordinates. J Chem Phys. 2016 Jun; 144(21):214108. https://aip.scitation.org/doi/abs/10.1063/1.4952956, doi: 10.1063/1.4952956.

[53] Parrish RM, Burns LA, Smith DGA, Simmonett AC, DePrince AE, Hohenstein EG, Bozkaya U, Sokolov AY, Di Remigio R, Richard RM, Gonthier JF, James AM, McAlexander HR, Kumar A, Saitow M, Wang X, Pritchard BP, Verma P, Schaefer HF, Patkowski K, et al. Psi4 1.1: An Open-Source Electronic Structure Program Emphasizing Automation, Advanced Libraries, and Interoperability. Journal of Chemical Theory and Computation. 2017; 13(7):3185-3197. https://doi. org/10.1021/acs.jctc.7b00174, doi: 10.1021/acs.jctc.7b00174, pMID: 28489372.

[54] Smith DGA, Burns LA, Simmonett AC, Parrish RM, Schieber MC, Galvelis R, Kraus P, Kruse H, Di Remigio R, Alenaizan A, James AM, Lehtola S, Misiewicz JP, Scheurer M, Shaw RA, Schriber JB, Xie Y, Glick ZL, Sirianni DA, O'Brien JS, et al. PSI4 1.4: Open-source software for high-throughput quantum chemistry. The Journal of Chemical Physics. 2020; 152(18):184108. https://doi.org/10.1063/5.0006002, doi: 10.1063/5.0006002.

[55] Mobley DL, Wagner J, Chodera J, Bannan C, Rizzi A, Camila, Bayly C, SimonBoothroyd, Lim NM, Lim V, Zhao Y, LeePing, openforcefield/openforcefield: 0.4.1 Bugfix Release. Zenodo; 2019. https://doi.org/10.5281/zenodo.3266364, doi: 10.5281/zenodo.3266364.

[56] Qiu Y, Smith DGA, Stern CD, Feng M, Jang H, Wang LP. Driving torsion scans with wavefront propagation. The Journal of Chemical Physics. 2020; 152(24):244116. doi: 10.1063/5.0009232.

[57] McGibbon RT, Beauchamp KA, Harrigan MP, Klein C, Swails JM, Hernández CX, Schwantes CR, Wang LP, Lane TJ, Pande VS. MDTraj: A Modern Open Library for the Analysis of Molecular Dynamics Trajectories. Biophysical Journal. 2015; 109(8):1528 - 1532. doi: 10.1016/j.bpj.2015.08.015.

[58] Wang LP, McKiernan KA, Gomes J, Beauchamp KA, Head-Gordon T, Rice JE, Swope WC, Martínez TJ, Pande VS. Building a More Predictive Protein Force Field: A Systematic and Reproducible Route to AMBER-FB15. J Phys Chem B. 2017 Apr; 121(16):4023-4039. https://doi.org/10.1021/acs.jpcb.7b02320, doi: 10.1021/acs.jpcb.7b02320.

[59] Development version of ForceBalance based on v1.6.0 used for 1.0.0 valence parameter fitting; 2019. https://github. com/leeping/forcebalance/tree/5b3a65d1baefe17625c9b05c0db5d27458c5344a.

[60] Lim VT, Hahn DF, Tresadern G, Bayly Cl, Mobley D, Benchmark Assessment of Molecular Geometries and Energies from Small Molecule Force Fields. ChemRxiv; 2020. doi: 10.26434/chemrxiv.12551867.v2.

[61] Ehrman JN, Bannan CC, Lim VT, Thi N, Kyu DY, Mobley DL, Improving Force Fields by Identifying and Characterizing Small Molecules with Parameter Inconsistencies. Zenodo; 2019. https://doi.org/10.5281/zenodo.3385278, doi: 10.5281/zenodo.3385278.

[62] Ehrman J, Lim VT, Bannan CC, Thi N, Kyu D, Mobley D. Improving Small Molecule Force Fields by Identifying and Characterizing Small Molecules with Inconsistent Parameters. ChemRxiv. 2020 Aug; doi: 10.26434/chemrxiv.12846602.v1.

[63] Sresht V, Rai B, PfizerRD torsional-strain, Calculation of Torsional Strain Energy; 2019. https://github.com/PfizerRD/ torsional-strain.

[64] Sterling T, Irwin JJ. ZINC 15 - Ligand Discovery for Everyone. Journal of Chemical Information and Modeling. 2015; 55(11):2324-2337. https://doi.org/10.1021/acs.jcim.5b00559, doi: 10.1021/acs.jcim.5b00559, pMID: 26479676.

[65] QCArchive Basic Examples, Optimization Datasets;. https://qcarchivetutorials.readthedocs.io/en/latest/basic examples/optimization_datasets.html/. 
[66] Frenkel M, Chiroco RD, Diky V, Dong Q, Marsh KN, Dymond JH, Wakeham WA, Stein SE, Königsberger E, Goodwin $\mathrm{AH}$. XML-based IUPAC standard for experimental, predicted, and critically evaluated thermodynamic property data storage and capture (ThermoML) (IUPAC Recommendations 2006). Pure and Applied Chemistry,. 2006; 78(3):541612. https://doi.org/10.1351/pac200678030541, doi: 10.1351/pac200678030541.

[67] Jorgensen WL, Chandrasekhar J, Madura JD, Impey RW, Klein ML. Comparison of simple potential functions for simulating liquid water. The Journal of Chemical Physics. 1983; 79(2):926-935. https://doi.org/10.1063/1.445869, doi: 10.1063/1.445869.

[68] Qiu Y, OpenFF "parsley" 1.0.0-RC2 release package; 2019. github.com/openforcefield/openforcefield-forcebalance/ releases/tag/v1.0.0-RC2.

[69] Jang H, Qiu Y, Openforcefield Parsley 1.0.0 Benchmark release package; 2019. https://github.com/openforcefield/ release-1-benchmarking/releases/tag/v1.0.0.

[70] Wagner J, Thompson M, Dotson D, Jang H, Rodríguez-Guerra J, Repository containing force fields released by the Open Force Field Initiative; 2020. https://github.com/openforcefield/openforcefields.

[71] OpenForceField, about/organization/; 2020. https://openforcefield.org/about/organization/. 